DOI 10.17816/transsyst20184477-111

\author{
(C) A. M. G. Jacob ${ }^{1}$, N. M. V. Monteiro ${ }^{2}$ \\ ${ }^{1}$ ISEP - Polytechnic School of Engineering \\ ${ }^{2}$ bimTEC - Building Information Modeling Consultants \\ (Porto, Portugal)
}

\title{
A NEW CONCEPT OF SUPERELEVATION IN MAGNETIC LEVITATION - PRODYNAMIC
}

Background: The topic of Magnetic Levitation systems, in terms of land mass transport, have created high expectations compared to aviation and also to the high speed railway industry. This new concept comes to revolutionize the terrestrial mass transport, in both the speeds and the subject of friction. Magnetic levitation solves the issue of attrition between material contact and as such may also be an opportunity to solve the question of constant physical superelevation.

Aim: Precisely that point of superelevation coupled with magnetic levitation, eliminating the rigid physical structures to laterally lift the vehicle in a curve. Current magnetic levitation systems do not address this issue of dynamic superelevation. It's exposed an improvement technology which is a theoretical possibility of a track through a new magnetic line can apply necessary rotation to the vehicle in curve and adjust its rotation according to the speed that vehicle moves.

Methods: In order to make this system to work it is suggested the introduction of a magnetic field in the new line, which will allow the vehicle to rotate in curves and will negate the need of the conventional static superelevation.

This study appeared as a result of an investigation of a master's thesis in civil engineering at ISEP, where the participants created the concept of dynamic superelevation in the context of magnetic levitation. The project was applied to the reformulation of an existing railway network. The study base of this model resulted from a broad survey of current magnetic levitation systems. Then came the idea of creating a third dynamic magnetic field to operate the curved superelevation.

Results: The result of the study was the creation of a new «monorail» system of simple and geometrically constant structure. The new line has the advantage of providing a simple and constant geometry, facilitating the manufacture, assembly and thus making it much more economical compared to the current systems. The cross-section allows the vehicle to fit perfectly and with the creation of rotating magnetic fields, the vehicle can be turned to both sides, at the required inclination, according the speed. With this new concept called ProDynamic, the geometry design in plan is totally independent of the speed practiced by the vehicle, where it can travel in curve at different speeds, but with the same lateral no-compensated acceleration, without detriment of passenger comfort.

Conclusion: Combining existing systems with this new concept, it is possible to create a total freedom in curves and superelevation, which will provide a maximum comfort and significant construction savings. There is therefore no longer a problem of deficiency or excess cant, as currently exists on railways. The advantage in the ProDynamic system is that it is possible to greatly reduce or even eliminate the lateral no-compensated acceleration.

Keywords: Levitation, Maglev, Train, High Speed Railway, Electromagnetism, Superelevation, Curve. 


\section{INTRODUCTION}

Magnetic levitation represents the first major advanced propulsion revolution since the creation of the internal combustion engine, in which this system offers the possibility of traveling faster, more economically and safer. The world is currently changing, the first electric vehicles are already emerging, and with these vehicles new ways of approaching transport, not only for road users, but also for railways. The railway is a known system and has evolved considerably but has limitations and drawbacks such as the lack of intrinsic friction of the wheel-rail contact system. The magnetic levitation system has the potential to transform and give rail transport a new dynamic by evolving high-speed lines in another dimension, shortening distances and being able to compete with aviation transport.

The first line of magnetic commercial levitation was recently implemented in Shanghai with the German Transrapid system, the Japanese government plans to have its magnetic levitation line operational in the next few years. In addition to these projects already in progress there are also plans for various countries. The scope of this article is a hypothetical approach to magnetic levitation systems, where it can be translated by the theoretical possibility of the vehicle turning on itself in a curve, thus not having to apply a static cant to the curved rail. This paper presents two different systems, which guarantee a higher productivity and have the objective of making the application of curved rails faster and faster. The curved application is equal to the application of the straight-line system. The only difference is that in curve there is a new magnetic field that induces the rotation of the vehicle. The rotation is performed by the track that rotates the vehicle in a corner at the angle of the speed of the vehicle, thus achieving a better relation between speed, rotation and comfort of the passengers.

This article describes the two new magnetic levitation systems proposed and idealized in the ISEP, which consist of the capacity of the vehicles to rotate on themselves, thus not necessitating the existence of superelevation in the rail. The characteristics of the track and the vehicle are also described, as well as the verification of the curve layout, the maximum possible slope for each system and the minimum curve radius to be implemented to guarantee the comfort of the passengers due to the non-compensated centrifugal force or even eliminate it.

\section{HISTORICAL CONTEXT OF MASSIVE LAND TRANSPORT}

The first rails to be created were rudimentary and go back to the early eighteenth century, where the first carriages were pulled by horses. With the 
industrial revolution, the development of railways has undergone a profound transformation.

Nowadays there are railway systems that can reach speeds more than $400 \mathrm{~km} / \mathrm{h}$, being systems that have evolved from the conventional railway line and have undergone some considerable adaptations to be able to move at high speeds.

Railways were also one of the great catalysts of the various disciplines of Civil Engineering, because due to the existence of natural and other geological obstacles, it forced the development of bridges, tunnels and other infrastructures. It also helped the growth of cities and their development as they were linked by a more efficient means of transport.

George Stephenson also built Locomotion 1, also known as The Rocket. It was the first locomotive with tubular boiler and with its own system of passenger transport called experimental. He was able to transport or pull loads weighing between 55 and $75 \mathrm{t}$ at a maximum speed of approximately $16 \mathrm{~km} / \mathrm{h}$. The following image shows a photograph of Locomotion 1 [2].

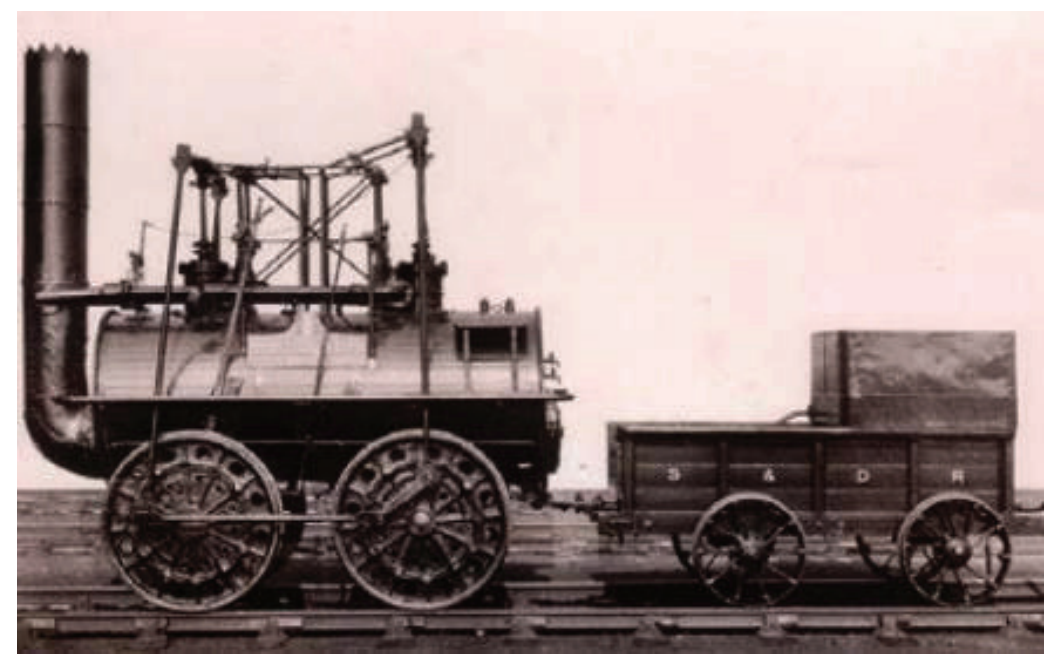

Fig. 1. Locomotion 1 "The Rocket” [1]

A new mode of transport was thus created as an exceptional growth rate that spread throughout the world and revolutionized the entire mass transport system.

The high-speed lines had their most significant development in the 1950s after World War II. On October 4, 1964, the first passenger high speed line was inaugurated by Japan Railway Shinkansen. The system implemented was able to reach the top speed of $210 \mathrm{~km} / \mathrm{h}$ and brought a return on investment in 7 years, having this train became known as a "bullet" train. The first minimum radius of the high-speed lines was $2500 \mathrm{~m}$, which was found to be too small and then increased to a minimum radius of $4500 \mathrm{~m}$, in a very short time. However, for speeds in the 
order of $300 \mathrm{~km} / \mathrm{h}$, again these spokes were too low. A minimum radius of $7000 \mathrm{~m}$ and preferably a minimum radius of $10000 \mathrm{~m}$ was then considered. The initial tunnel section was $62 \mathrm{~m}^{2}$ and for the new "bullet" trains the current minimum section is about $100 \mathrm{~m}^{2}$.

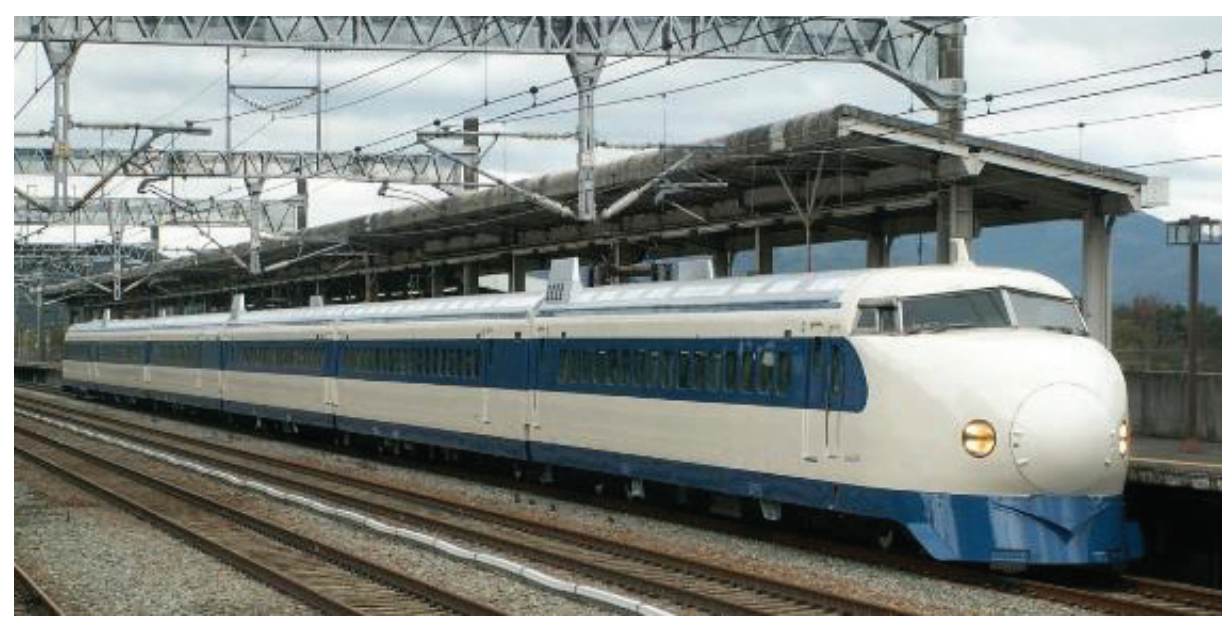

Fig. 2. Highspeed Train JP Shinkansen S-0 (Source: ナダテ Nadate, 2008)

At the beginning of the new $21^{\text {st }}$ century, JR Shinkansen began to develop the third generation of high-speed railway lines, with the purpose of increasing the speed of operation to $350 \mathrm{~km} / \mathrm{h}$. Thus they conceived the E5 in a program called Fastech 360 capable of reaching speeds of $320 \mathrm{~km} / \mathrm{h}$ as can be seen in next image [4].

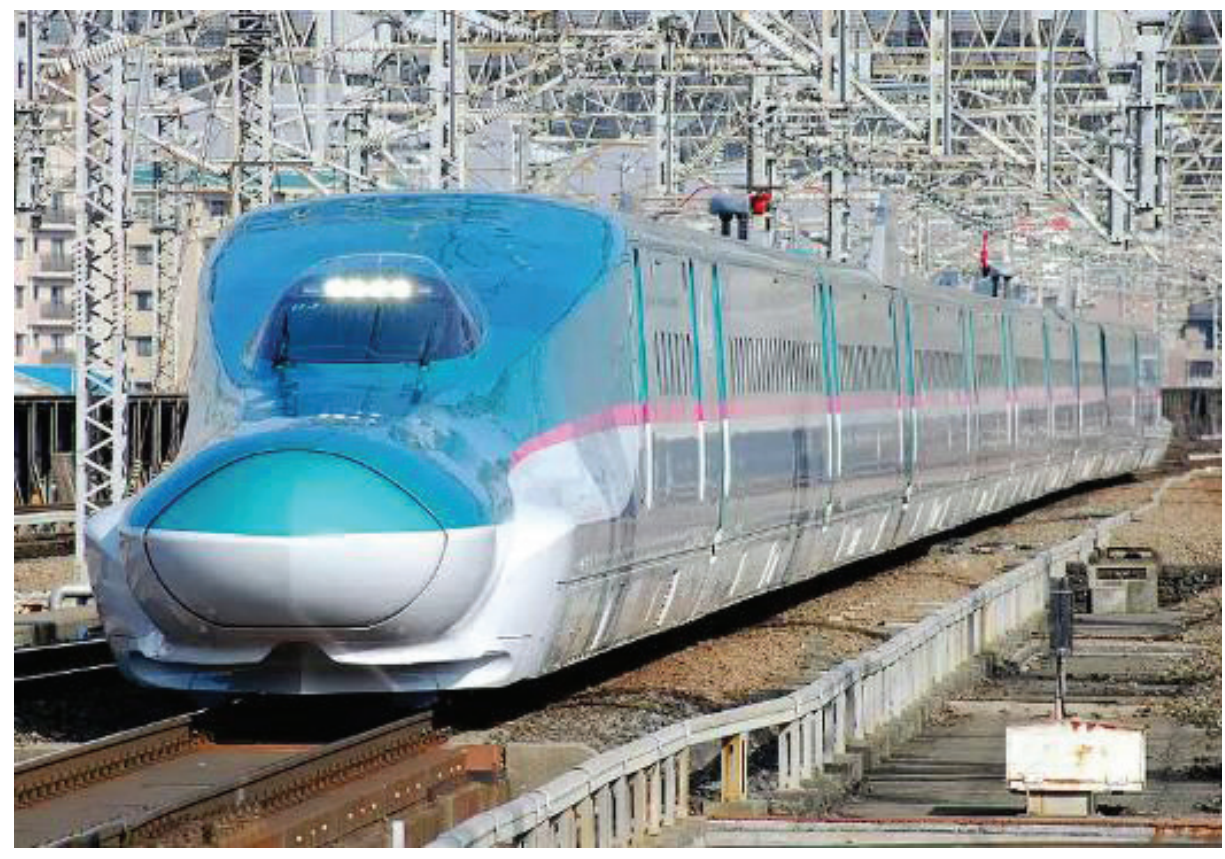

Fig. 3. Highspeed Train JR Shinkansen E5 (Source: Nanashinodensyaku, 2011) 
The second country that successfully developed the high-speed railway was France. It built the first high-speed line from Paris to Lyon using TGV-PSE technology in 1981, 17 years after Japan. According to the classification, these trains were able to reach speeds upper than $280 \mathrm{~km} / \mathrm{h}$, belonging to the technology of first generation. Second-generation trains appeared 20 years later, on the TGVMediterranée line, from Valence to Marseille, with an upgraded infrastructure. In 2007, the new TGV-EST high-speed line from Paris to Strasbourg was completed. Both lines were able to provide better conditions for the movement of the train and the maximum speed of operation of the TGV-Mediterranée and TGV-EST, thus increasing up to $320 \mathrm{~km} / \mathrm{h}$, with France reaching the speed of $300 \mathrm{~km} / \mathrm{h}$ trains of the first generation. The French TGV also had the highest operating speed of the second generation, $320 \mathrm{~km} / \mathrm{h}$, which is the world record by 2008. In the third generation of high-speed, France realized that the centralized power system they used to the first generation and the second generation was not suitable for speeds above $350 \mathrm{~km} / \mathrm{h}$. Therefore, they adopted the existing technology in Japan with the distributed power system, designing their third-generation train, the AGV360, with the fastest train in the world, reaching a speed of $574.8 \mathrm{~km} / \mathrm{h}$ on April 3, 2007.

However, the operating speeds of these systems are around $300 \mathrm{~km} / \mathrm{h}$ for track maintenance, such as ballast, crossbars and anchorages, as well as track wear. That is, at this moment, the speed limitation in the railway is around $320 \mathrm{~km} / \mathrm{h}$, due to safety and especially maintenance/wear, taking into account that this system has permanent physical contact between two materials.

To overcome this major problem of wear/safety, a new system emerges that can be considered a fourth-generation system reaching operating speeds of over $430 \mathrm{~km} / \mathrm{h}$. This new system uses magnetic levitation to move and levitate on the rails, and which is now in commercial use in Shanghai in China, proving to be a success and promising for the future.

Aviation can be the most competitive means of transport, for distances greater than $1500 \mathrm{~km}$, when compared to high-speed railway. However, with

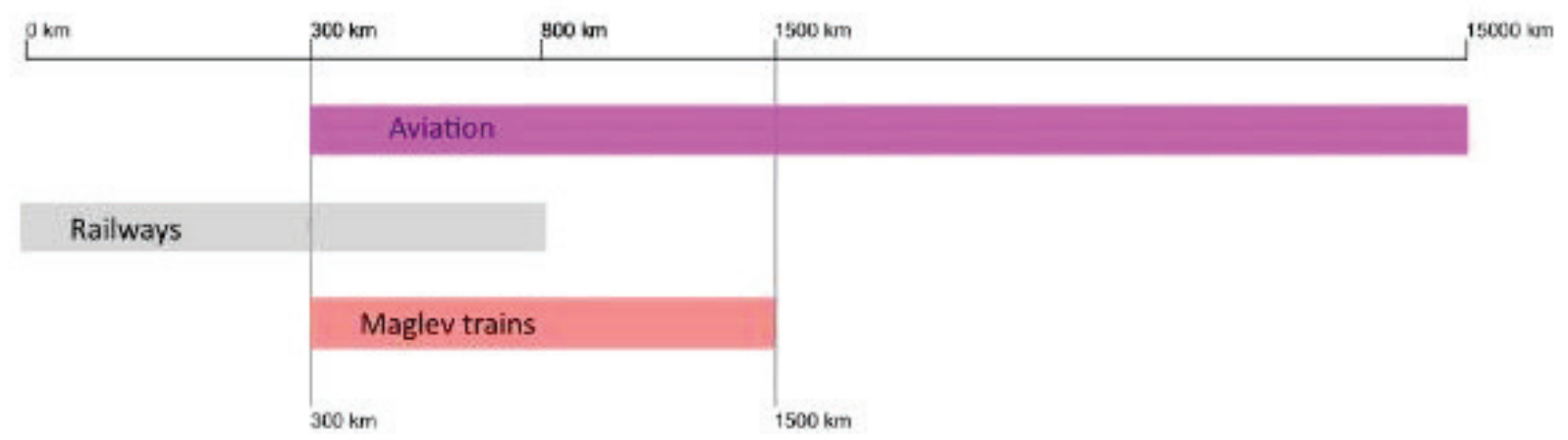

Fig. 4. Comparison of means of transport taking into account the economic factor 
magnetic levitation, for speeds between 450 and $600 \mathrm{~km} / \mathrm{h}$, it could be a competitive option to aviation, up to distances of $2000 \mathrm{~km}$. In conclusive terms, there is a very competitive space between high-speed railway and aviation, which can serve very well magnetic levitation, between $300 \mathrm{~km}$ and $1500 \mathrm{~km}$.

\section{HISTORICAL REFERENCES OF MAGNETIC LEVITATION}

Maglev levitation uses magnetic forces to lift, orient and propel vehicles, using both attractive and repulsive forces. At the beginning of the $20^{\text {th }}$ century, Emile Bachelet designed a magnetic suspension that used repulsion forces generated by alternating current. This concept of Bachelet was impracticable for the time because the amount of energy of the conventional conductors was insufficient. It was only in the 1960s when superconducting magnets emerged that it became possible to develop this technology. In the early 1920s, the work of Hermann Kemper in Germany pioneered a Maglev using attraction, called attractive-mode maglev. Kemper explored this concept during the 1930s and in the 1940s established a basic design to implement his Maglev system, having published an article in 1953.

In 1963, scientists James R. Powell and Gordan T. Danby patented the first Maglev system using superconductivity, a system based on the work of Emile Bachelet, overcoming the energy limitations that underlie this technology. In 1966, they introduced their concept of Maglev using superconductors in the vehicle and coils on the sides of the rails. This technology was then followed by the system used in the Japanese Maglev.

Another important work using Maglev technology was carried out in the United States of America in the early 1970s and refers to the development of ROMAG (people-mover demonstration vehicle) by Rohr Corporation. This system was normally driven by electromagnets that generate attractive forces between the vehicle and ferromagnetic material in the rail, a system designated by magnetic suspension system (EMS). Unlike EDS, the EMS is statically unstable, a control system that varies the currents in the electromagnets to maintain clearances between the vehicle and the lane.

The unit of measure of the International system that measures magnetic flux density is the Tesla, and thus was instituted in honor of Nikola Tesla at the Conférence Générale des Poids et Mesures, Paris in 1960. Nicola Tesla was an inventor in the field of mechanical engineering and electrotechnology and the creator of the alternating current that exists today in all the electrical infrastructure.

Magnetic levitation is defined as the suspended state of a body in space at some distance from the surface. To achieve this state of suspension, magnetic forces that compensate for the force of gravity are used. The magnetic field exerts 
a force strong enough to levitate and stabilize the body at a certain position relative to the surface.

In March 1912, the engineer and inventor Emile Bachelet was able to register a patent called "Levitated Transmitting Apparatus", showing the New York public a model of a Maglev train, as can be seen in next figure.

One of the first applications of magnetic levitation was developed by Gene Covert and his MIT colleagues who created the magnetic suspension and balance system.

Maglev technology was first proposed by Emile Bachelet. A few years later, Werner Kemper proposed a type of magnetically levitated train. He obtained the patent for the magnetic levitation train on August 14, 1934 [11]. The evolution of his patent resulted in the creation of the magnetic levitation system currently used on Transrapid.

Another Maglev system with commercial use is the HSST (High Speed Surface Transportation) system, is the Japanese system that in 2005 started its commercial use called Linimo. It is an urban system with a maximum speed of $100 \mathrm{~km} / \mathrm{h}$. It is identical to the system used in Transrapid, using the attractive forces to move in the line, distinguishing itself from the Transrapid by the maximum speed that each vehicle can reach and by the differences that exist in the linear motors [13].

The development of magnetic levitation technology under the "railway" lines had different evolutions that led to different solutions of magnetic levitation.

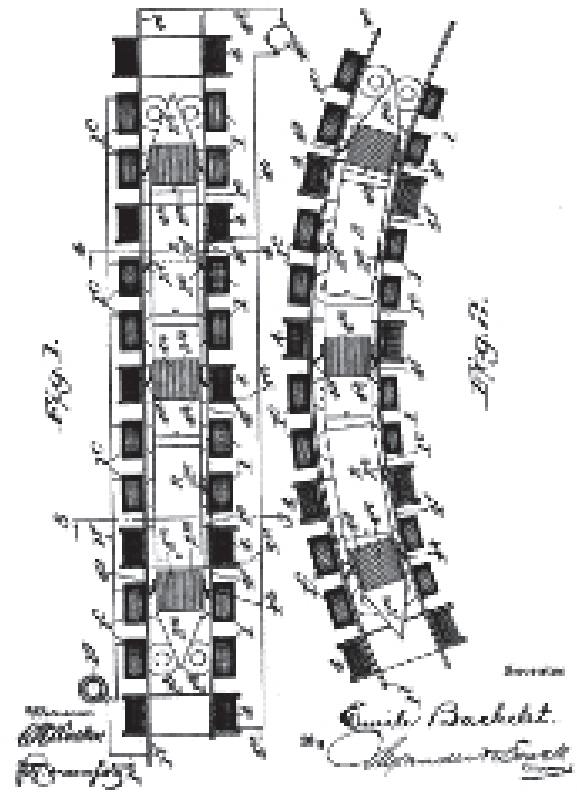

Fig. 5. Emile Bachelet system patent image [11]

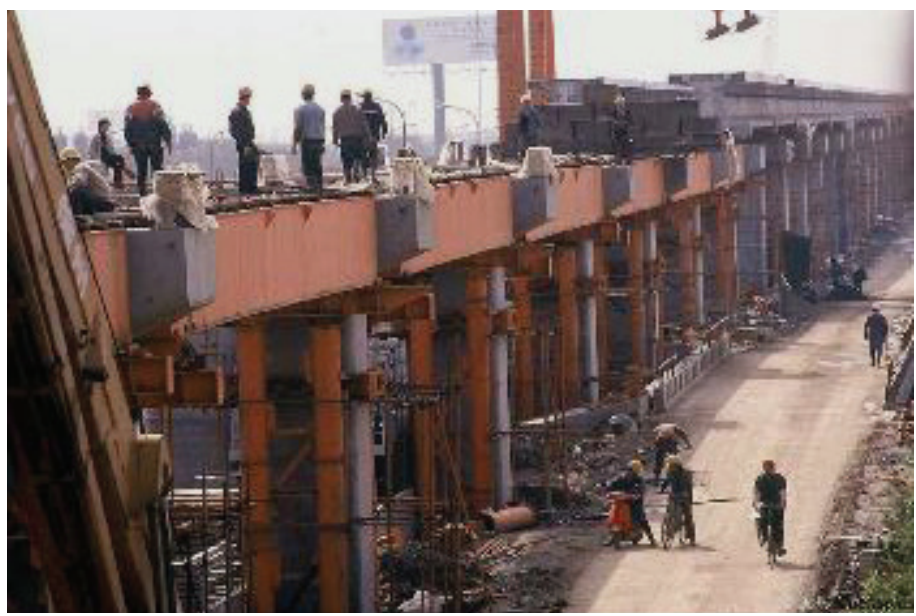

Fig. 6. Construction of Transrapid infrastructure in Shanghai [12] 


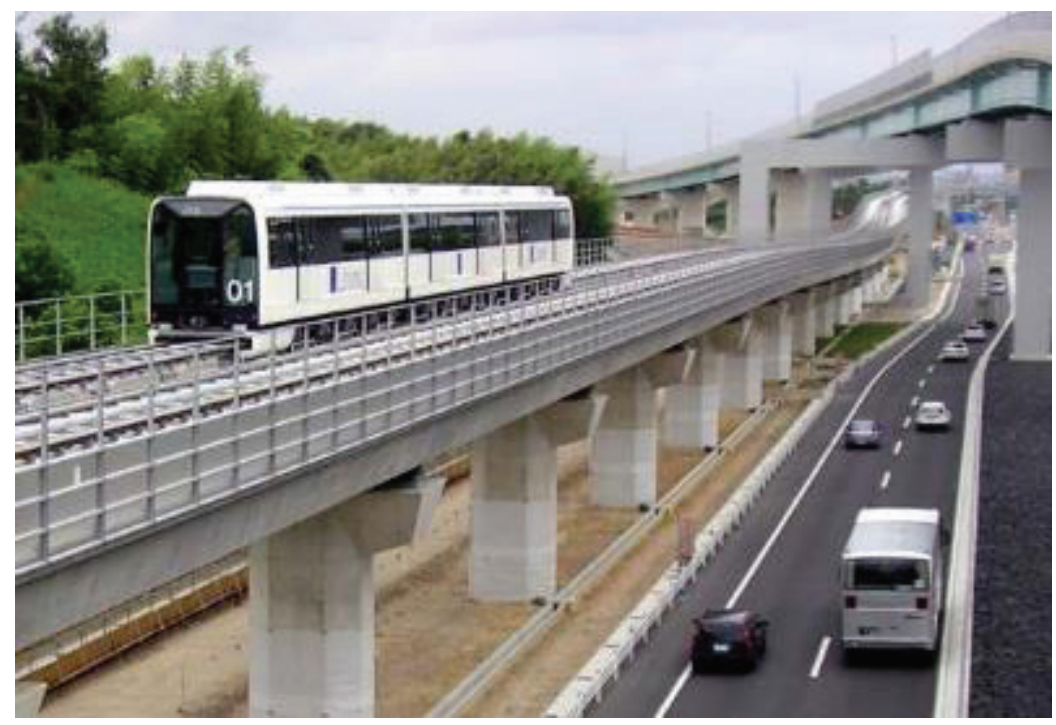

Fig. 7. Urban Maglev system Linimo [13]

Different types of magnetic levitation technologies have been achieved, with three technologies currently under development and some already in commercial use, such as the German Transrapid. Systems currently under development are EDS, EMS and INDUCTRACK.

The designation EMS comes from Electromagnetic Suspension, being the system used in Transrapid. It concerns the forces of magnetic attraction for levitation. This system uses electromagnets that are controlled electronically and individually. This type of levitation is by nature unstable and requires an effective control system with great precision to keep the Maglev train suspended.

The EDS system stands for Electrodynamic Suspension and is used by the Japanese in the JR SCMAGLEV (Japan Railway). It refers to the magnetic repulsion that consists of the use of superconductive coils that create repulsive forces propelling the vehicle. The coils are located inside the train that create a strong magnetic field in connection with the ferromagnetic bars on the rails that levitate the vehicle.

The INDUCTRAK system is the latest magnetic levitation technology. It does not use superconducting magnets or electromagnets, but permanent magnets at ambient temperature, like ordinary but more powerful magnets. These magnets produce an oscillating magnetic field and, consequently, levitate the vehicle. The main advantage of Inductrack technology over the others is that, because it is an induction activated repulsive force system, it is stable and control circuits are almost unnecessary to achieve stability.

In the 1970s, the Germans and Japanese began to develop their versions of Maglev technology. The Japanese, supported by the Japanese National Railway 


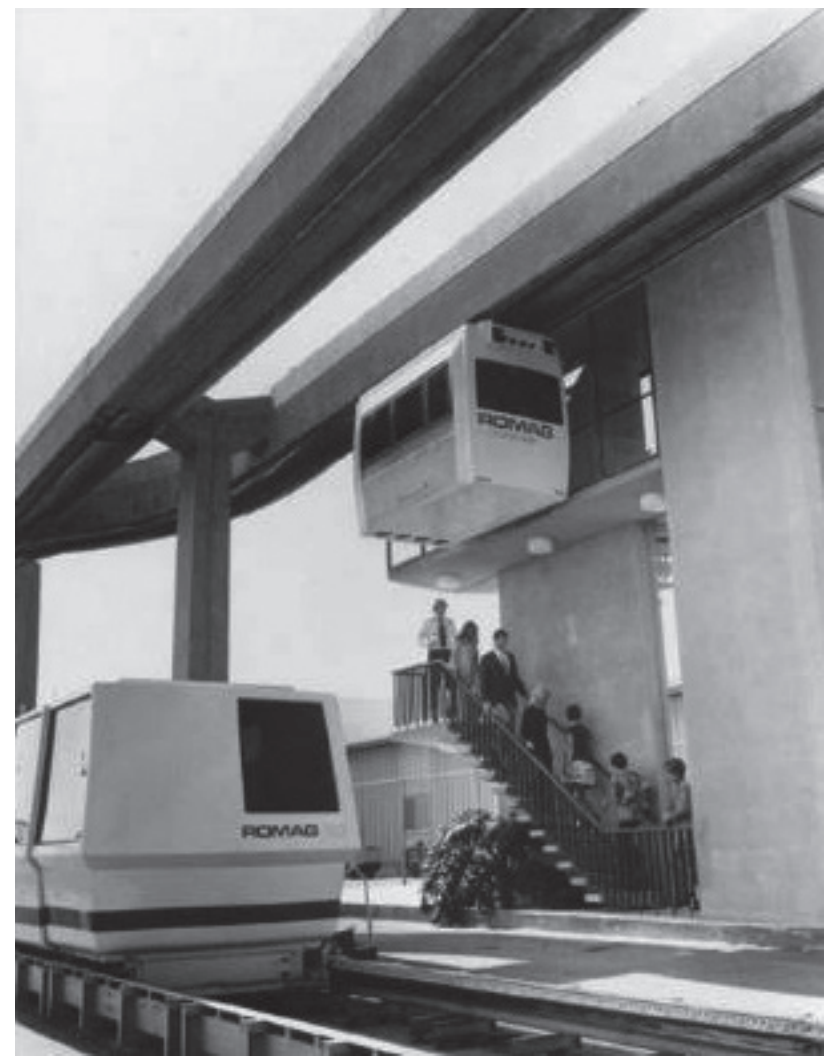

Fig. 8. ROMAG Maglev [10]

(JNR), have expanded the development of their Maglev technology. By 1972, they had already built their model, having in 1974 their large-scale runway.

In 1970, in Germany there were already some prototype vehicles operating under the German government's jurisdiction, these being the first versions of the current Transrapid [10].

In 2003, the first commercial line with the Maglev Transrapid system with $30.5 \mathrm{~km}$ length is finally opened in Shanghai taking the time of $7 \mathrm{~min} 22 \mathrm{~s}$.

\section{MAGNETIC LEVITATION SYSTEMS}

Magnetic levitation trains have several advantages compared to the conventional rail system because it is a system where there is no contact with the rail, so there is no friction between materials without physical wear.

There are already several studies showing that magnetic levitation systems produce less noise (about $85 \%$ less) compared to high-speed railway systems.

They are also able to overcome greater longitudinal inclinations compared to the railway system (about $10 \%$ inclination up to $12 \%$ ), while on the railway the maximum is about $2.5 \%$ (commonly characterized by $25 \%$ ). 
Levitation systems achieve high speeds over a shorter distance when compared to high-speed railway systems. Magnetic levitation vehicles can achieve better acceleration and braking performance over shorter distances than the highspeed rail, about $1 / 5$ of the stroke length.

The magnetic levitation system is more secure compared to high-speed railway systems because it is almost impossible to «derail» due to the way the vehicle is gripped and envelops the rail.

Magnetic levitation lines have a considerably reduced operating cost compared to rail lines around $60 \%$ less, due to the low energy consumption of the system, to relatively low wear. However, construction costs are more expensive for Transrapid by around $60 \%$ compared to ICE [3, 14].

The vehicles of the conventional rail system transmit their weight to the rail through the axles, these being punctual loads and very high. The Maglev transmits a uniformly distributed load to the «rail», which results in less punctual efforts. In addition, it should be noted that the Maglev vehicle is considerably lighter than the conventional rail vehicle because it has no wheels or engines, less metal mass. It can be said that for the construction of an infrastructure where a vehicle with Maglev technology circulates, this structure will be slimmer and more economical.

The tunnel sections required for the Maglev system may be smaller compared to the existing sections in the rail system, ensuring economy and speed of construction.

The minimum radii of the curves for the Maglev system are smaller, thus translating a more efficient layout and less area of land to be expropriated [15].

Thus, it can be stated that although the Maglev system is about $60 \%$ more expensive in implementation, this being the only unfavorable aspect of this technology, all other aspects are great advantages for the Maglev system. Electric consumption and the emission of greenhouse gases are intrinsically linked, and this system guarantees a reduced electric consumption compared to the current rail system, also guaranteeing greater energy and environmental sustainability in this area of transport.

\section{EMS SYSTEM}

The EMS system results in the culmination of an arduous and concentrated research in Germany over fifty years of a technology that originated in the United States of America. It is an extremely sophisticated and secure electronic integral control system [5]. The next image represents the Transrapid system already in commercial use. 


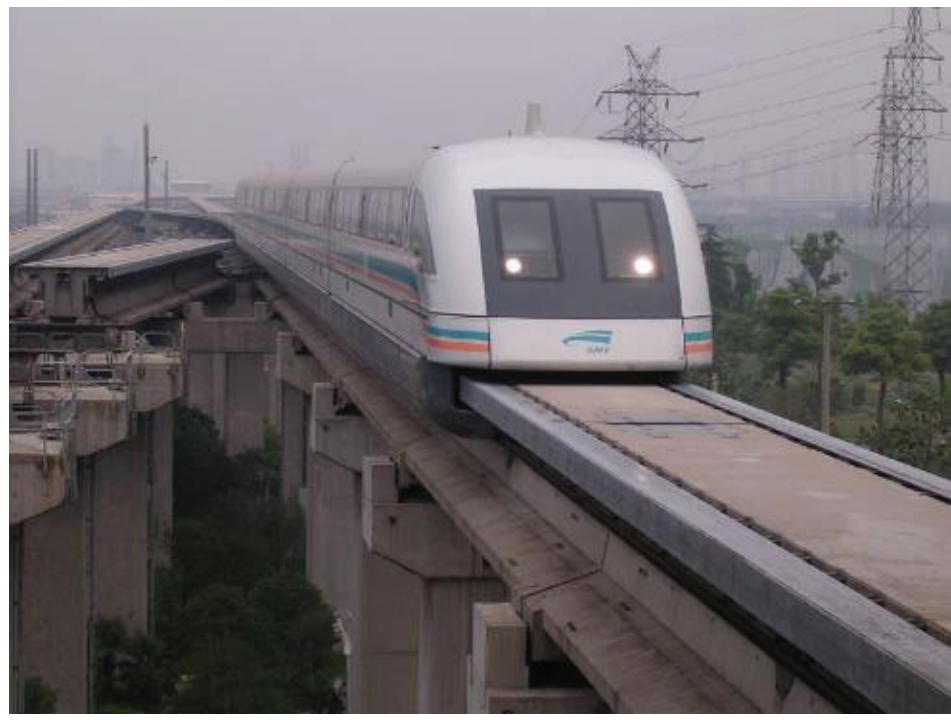

Fig. 9. EMS technology used in Transrapid (Source: Siemens AG Transportation Systems, 2005)

This system emerged in the 1970s when Transrapid's German trains used the pulling forces between electromagnets and ferromagnetic bars that are controlled individually and electronically and levitate the vehicle. These electromagnets are in the vehicle and the ferromagnetic bars of the rails. The next image demonstrates a scheme of the levitation system used in Transrapid.

The vehicle has a grip-shaped bracket that involves the lateral rail where the electromagnets are located and these pull forces on the ferromagnetic supports that are located under the rails ensuring lift and levitation of the vehicle. Laterally there is a set of electromagnets that serve as a lateral guide, which has the function of controlling the transverse movements of the vehicle on the rail, thus ensuring

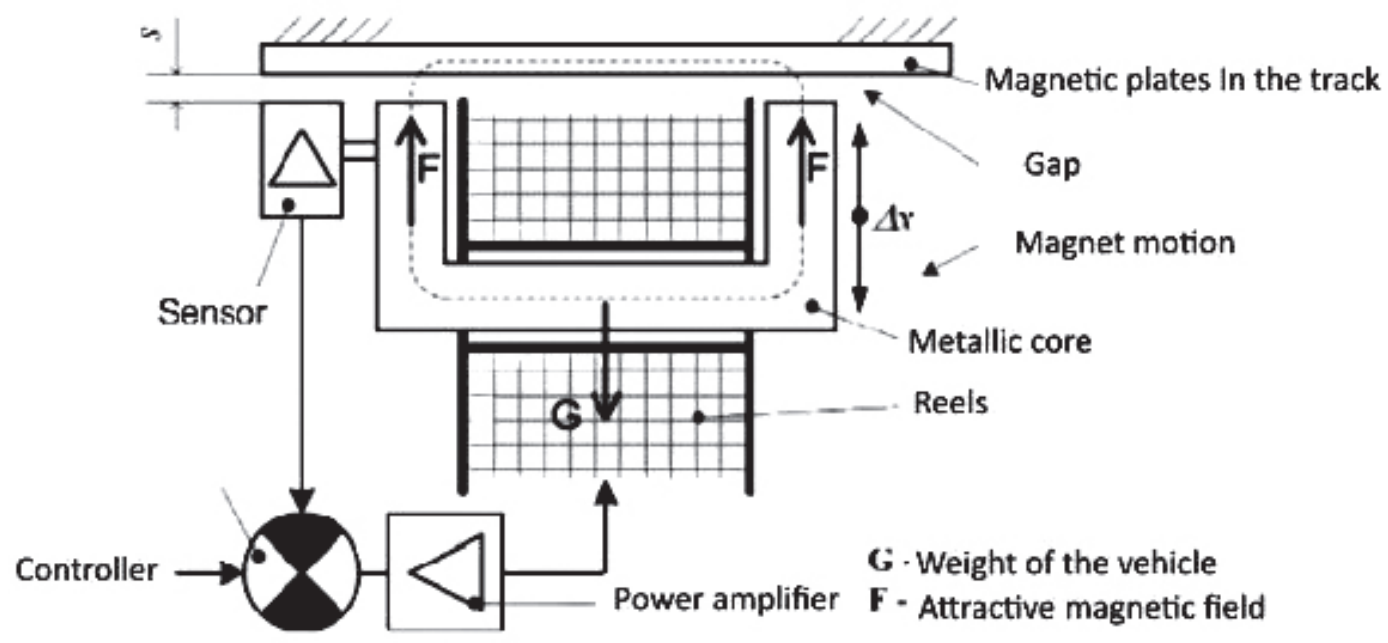

Fig. 10. Principle of Levitation EMS [3] 
that the vehicle is centered in the track while traveling. The distance between the sides of the vehicle and the rails on the sides is $10 \mathrm{~mm}$, and this is controlled electronically, while the distance between the rail and the bottom of the vehicle distance is $150 \mathrm{~mm}$, which makes it possible to go over small objects or small layers of snow [5]. The nest figure shows a representation of the Transrapid system.

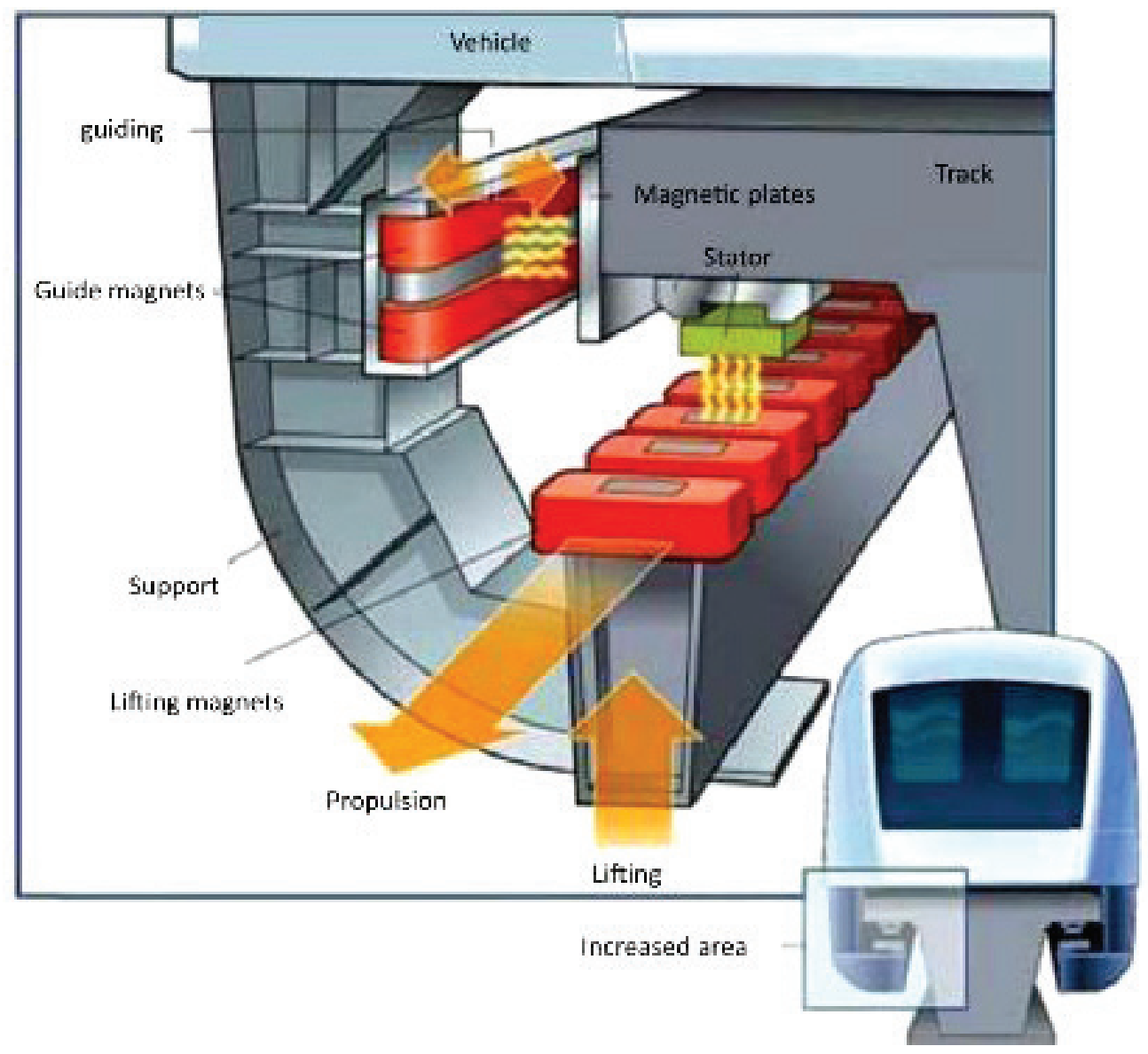

Fig. 11. EMS system used in Transrapid [6]

The propulsion system used is the synchronized linear stator motor, which is used for the propulsion and braking of the vehicle. This motor is also referred to as LMS - linear motor stator. This motor thus creates a magnetic field, which when interacting as the support magnets in the vehicle arm create forces of attraction impelling the movement of the vehicle. The magnetic field is achieved by induction of a three-phase alternating current, the speed of the vehicle is controlled by the frequency of that same alternating current of the linear motor. Braking the vehicle is achieved by changing the direction of the magnetic field, there being no physical contact between the two sides of the engine. The linear long stator motor installed in the rails is divided into sections, these being fed by the electric current only 
when the vehicle is in the section in question. To do this, substations with different powers are required according to the track layout, since they can be traced that require considerable braking and large accelerations of the propulsion system, a situation that requires a greater power of the electric network, compared to the traces of the track in which the journey is constant.

Shown in the next figure is a diagram of how the chain moves and feeds the vehicle along the rails. It is verified that there is power for each section of rail.

The EDS (Electrodynamic Suspension) system is used in the Japanese Maglev, it uses magnetic repulsion as opposed to the EMS system, this system uses superconducting coils that give rise to repulsive magnetic forces causing the vehicle to move. The vehicle needs wheels, because at low speeds the vehicle can not reach levitation, which can only be achieved after the vehicle reaches a speed of more than $120 \mathrm{~km} / \mathrm{h}[6]$.

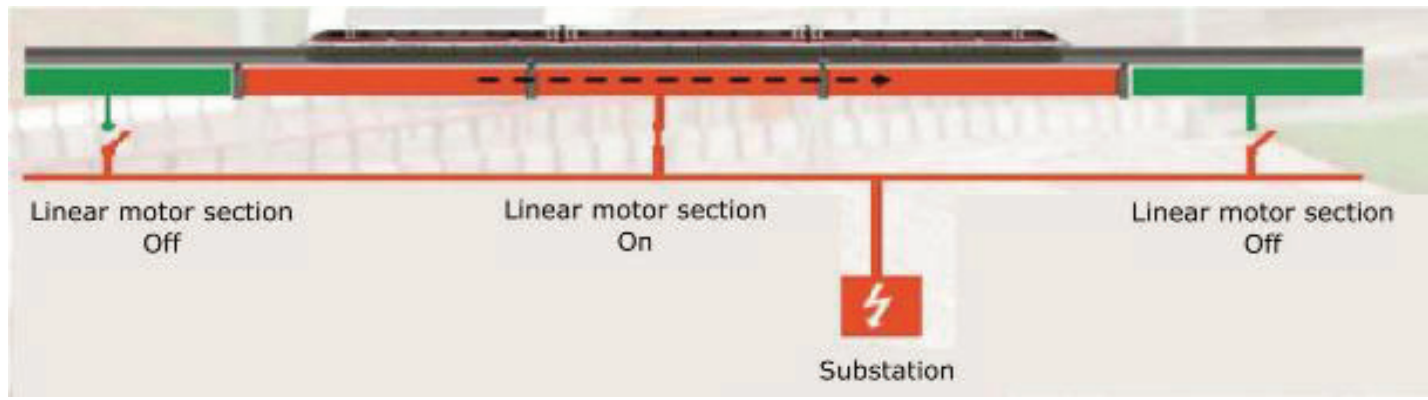

Fig. 12. Linear motor sections on track [7]

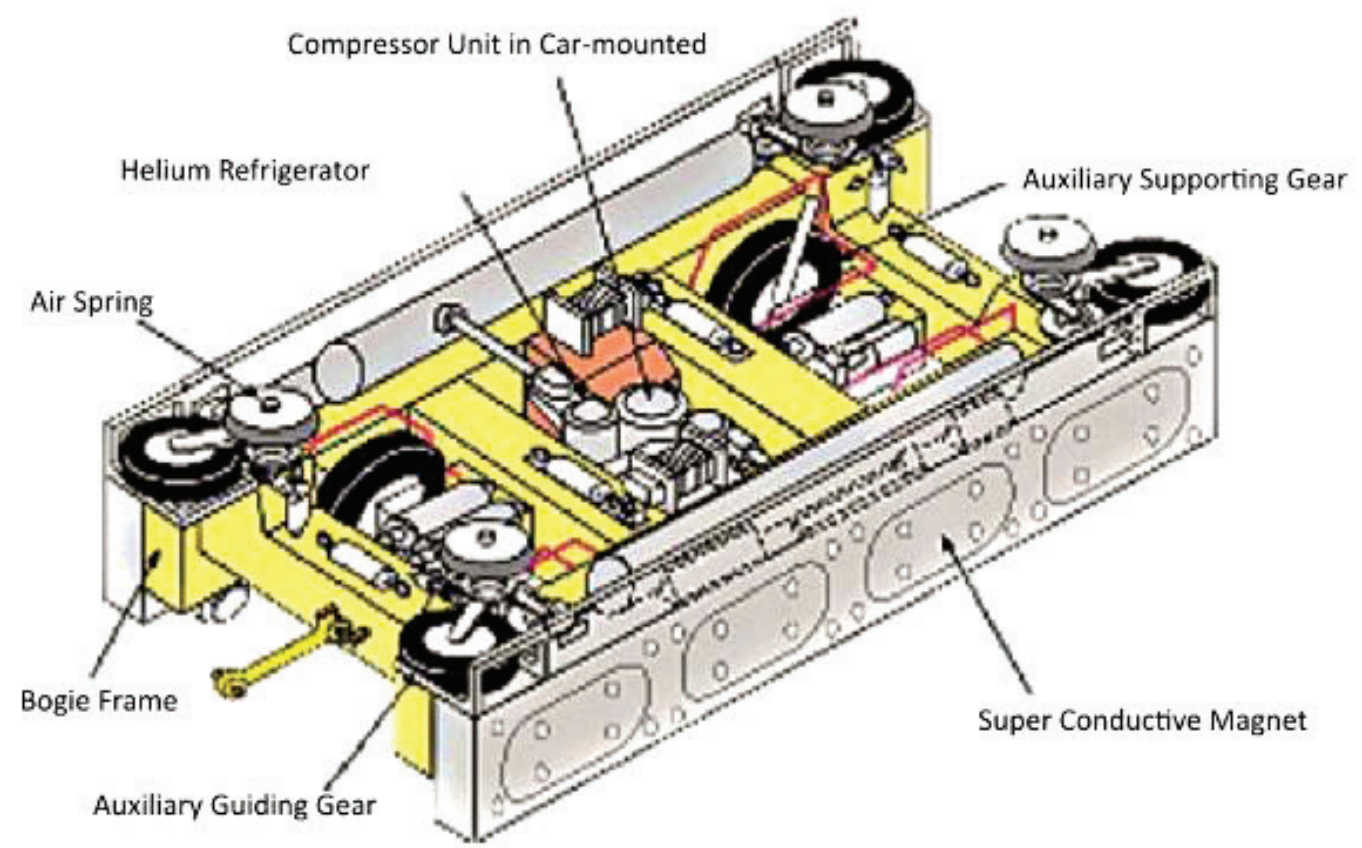

Fig. 13. MXL01 bogie system (Source: Odec.ca, HBLee, 2007) 
The EDS is based on the repulsive forces of lorentz, resulting from the interaction of the superconducting coils in motion with the existing coils aluminum rails. The next figure shows the MLX01 system of the Japanese magnetic levitation system in the Yamanashi test line.

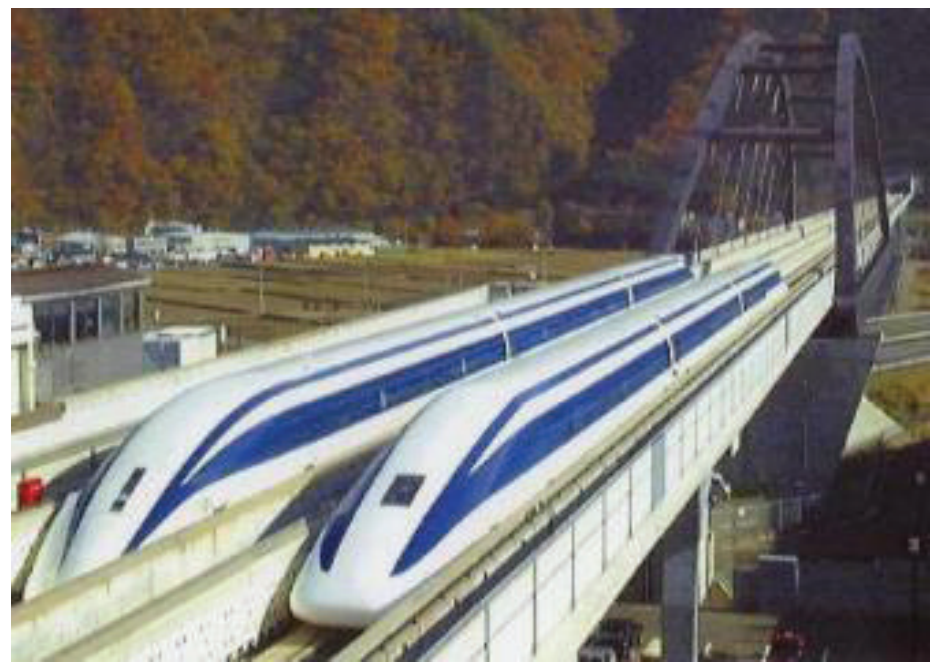

Fig. 14. EDS technology used in the JR-Maglev MLX01 Japanese System (Source: Central Japan Railway Company - Yamanashi Maglev Test Line, 2000)

The interaction between the superconducting coils in the vehicle and the coils on the rail creates a strong magnetic field that is induced by the superconducting coils of the vehicle, which causes the coils on the rails to undergo polarity inversion, creating repulsive forces that levitate the vehicle. The coils on the rails are passive, non-superconducting, and have an " 8 " configuration, which causes the current induced by the coils in the vehicle to cause a simultaneous force that pulls the vehicle upward and pushes it down.

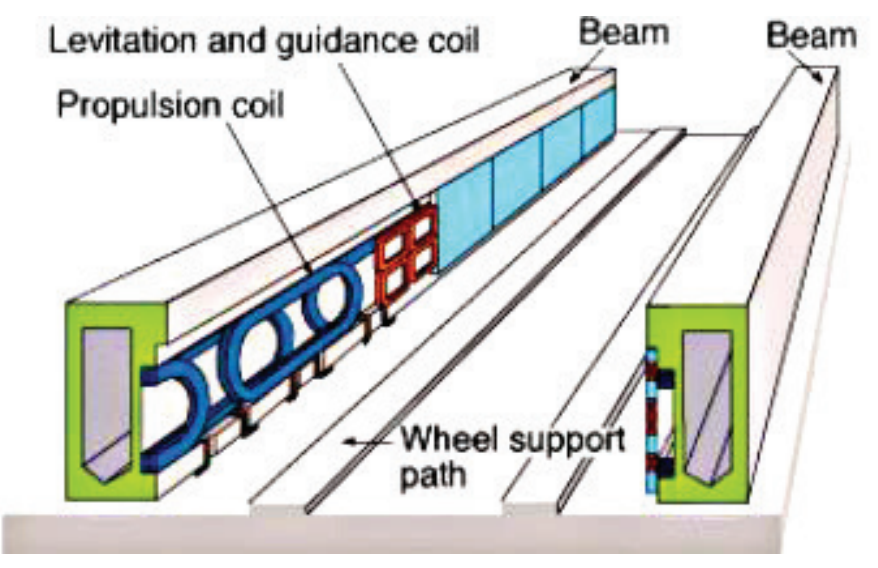

Fig. 15. Schematic of the rail used in JR-Maglev MLX01 [6] 
The EDS system used in the JR-Maglev MLX01 uses two types of coils that have different functions on the sides of the rails. They have the function of guiding and propelling the vehicle on the track and in addition to these reels, the rail has a support for the wheels, which the MLX0 uses when traveling at speeds below 120 $\mathrm{km} / \mathrm{h}$, as shown in next figure $[6,8]$.

The propulsion coils located on the sides of the rails are fed by a three-phase electric substation, creating the polarity variation that will give rise to the magnetic field that drives the vehicle when they come into contact with the superconductors in the vehicle. The movement of the vehicle is carried out by alternating polarity in the lateral coils of the rails, as can be seen in next figure. The lateral coils on the left and right side of the rail alternates the polarity, thus provoking forces of attraction or repulsion forces, in the superconductors on the side of the vehicle, impelling the movement $[6,8]$.

Inductrack was developed in the 1990s by Dr. Richard Post at Lawrence Livermore National Laboratory in California. The system uses conventional magnets in Halbach Array, that is, Halbach arrangement, and to achieve levitation, these conventional magnets are arranged in a certain way creating a very specific magnetic field known as the Halbach arrangement. These thus create levitation,

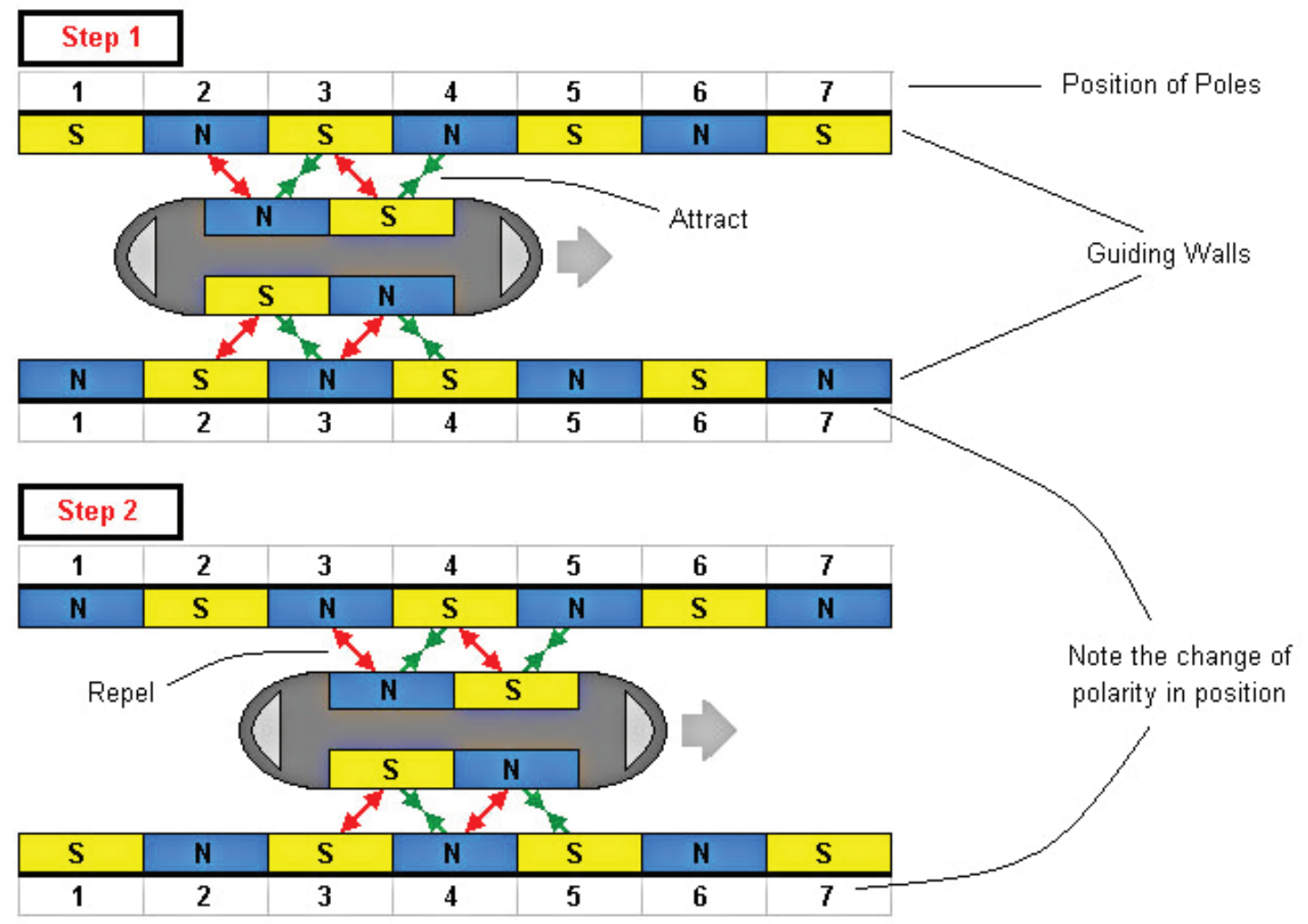

Fig. 16. Diagram of operation of the EDS System and how to change the polarity (Source: Fiyaz Ahmed, 2009) 
unlike the other Maglev technologies used by the Japanese in the EDS and by the Germans in the EMS, which rely on sophisticated sensors and cryogenic refrigeration systems, to ensure levitation and movement of the vehicle on the rails. In addition, the magnets are composed of a new material comprising a neodymiumferro-boron alloy, which generates a higher magnetic field.

The main piece of technology is the Halbach Arrangement, which is what defines the Inductrack system and distinguishes it from other competing Maglev technologies. The Halbach Arrangement guarantees some benefits that include increased safety and efficiency and reduced cost. This system was developed in the 1980 s by physicist Klaus Halbach, which consists of a set of conventional magnets placed in a series of repetitions and with specific orientations that create a strong magnetic field. The following image illustrates how the magnetic field lines override the magnetic field above and at the same time create a strong magnetic field beneath the magnets. The Halbach Arrangement induces an electric current in the levitation circuits consisting of Litz wires thus levitating vehicle.

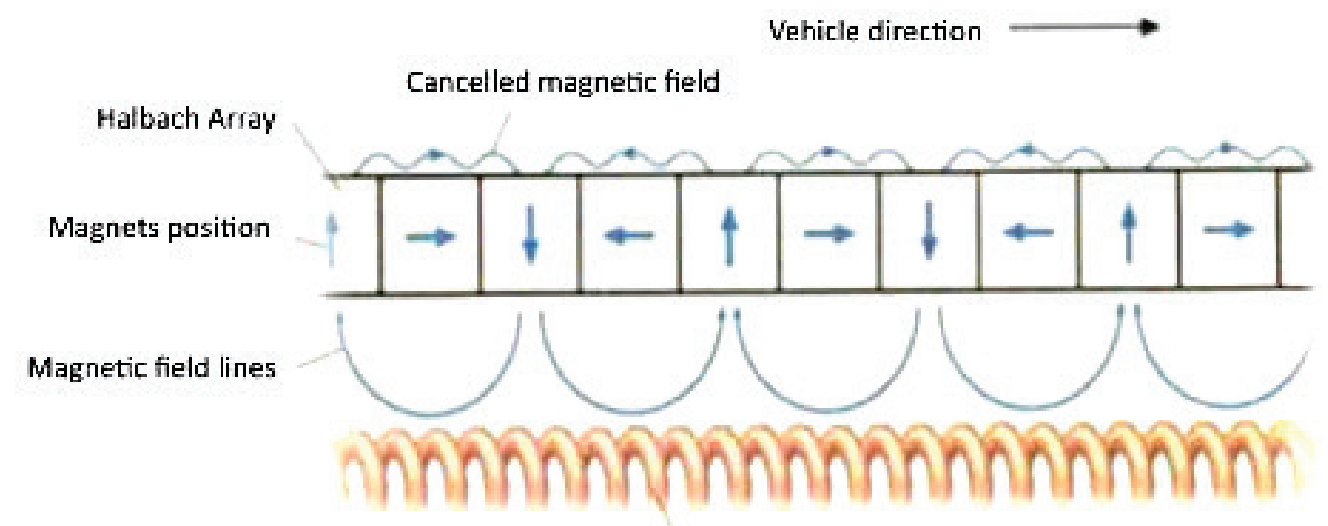

Levitation circuits

Fig. 17. Schematic of the magnetic field formed by the Halbach Array [16]

In this system there is still no comercial prototype built on a real scale because it is a recent system, but the experiences with this technology reveal many potentialities. In the next figure you can check the test vehicle that was built [16].

This vehicle with the Inductrack system when it is built will require four Halbach Arrangements, over a series of levitation circuits consisting of Litz wires. Two of the Halbach Arrays are positioned down over the circuits if they have the levitation function while the other two Halbach Arrays are positioned sideways in relation to the circuits to balance the vehicle to maintain it, as it can be seen in next image [16].

The effects of the Halbach Arrangement were substantiated by extensive tests done by Dr. Richard Post, the inventor of Inductrack, which tried to test their initial calculations, which suggested that the Halbach Arrays could levitate loads 


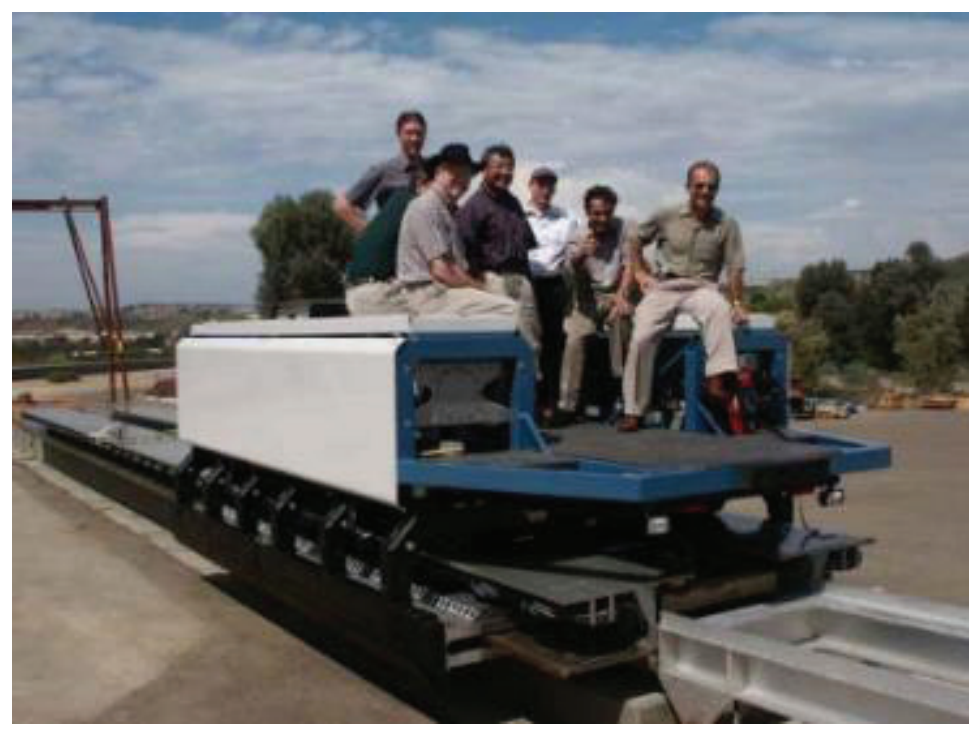

Fig. 18. Inductrack experimental vehicle [9]

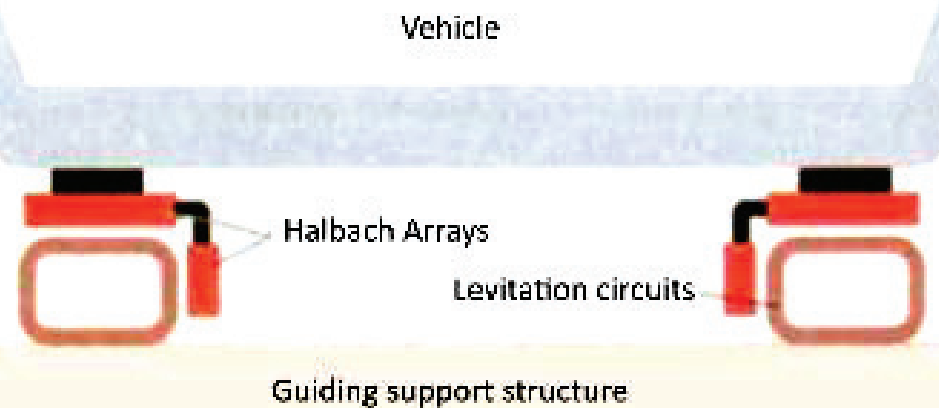

Fig. 19. Representative scheme of a possible vehicle with the Inductrack system [16]

weighing up to fifty times the weight of the magnets. To test this hypothesis, the scientific team built a scale model and launched it at a speed of about $35.4 \mathrm{~km} / \mathrm{h}$. The model proved to be stable and the lifting force proved to be that verified in the theory [16].

\section{COMPARISON BETWEEN DIFFERENT TECHNOLOGIES EMS, EDS, INDUCTRACK}

Currently there are three known Maglev systems, the electrodynamic suspension system (EDS), the Electromagnetic Suspension System (EMS) and Inductrack. Each of the three systems has different characteristics and very specific characteristics. While EDS and EMS use only the interaction of magnets and 
superconductors, the Inductrack uses coils in rails underneath vehicle, however the three suspension systems work under the same principle of magnetic levitation. The following image demonstrates the three existing levitation systems.
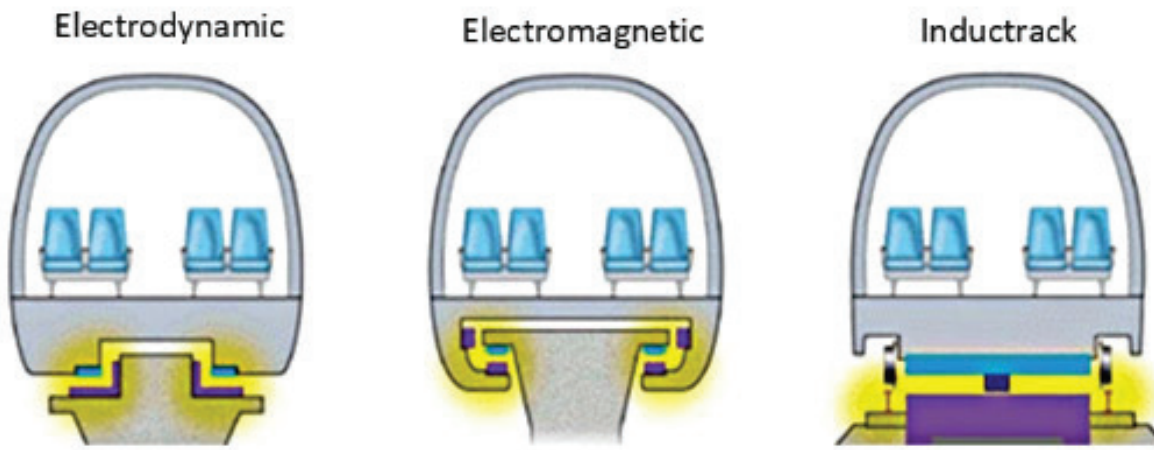

Fig. 20. Comparison systems between Magnetic Levitation Systems (Source: Maglev Trains A Look into Economic Concessions, Binyam Abeye, 2011)

\section{The EMS system used in Transrapid has the following advantages:}

- The magnetic fields of the system inside and outside the vehicle are smaller than in the EDS system;

- It is a commercially available technology that can reach high speeds, about $500 \mathrm{~km} / \mathrm{h}$;

- No auxiliary wheels or secondary propulsion system needed;

- The configuration of the vehicle in the form of grabs (locks) makes it almost impossible to derail the vehicle;

- The noise is due only to the displacement of the mass of air caused by the locomotion of the vehicle.

\section{The disadvantages of the EMS System are:}

- The gap between the vehicle and the guide rail must be constantly monitored and corrected by computer systems to avoid collision due to the unstable nature of electromagnetic attraction and possible irregularities in the rail;

- More vibration problems may occur.

The EDS system used by MXL01 has the following advantages:

- Superconducting magnets in the vehicle allow high speeds of around $600 \mathrm{~km} / \mathrm{h}$;

- Ability to carry heavier loads;

- Successful operations with the use of high-temperature superconductors on your liquid helium cooled magnets.

\section{The disadvantages of the EDS System are:}

- Intense magnetic fields due to superconductors on board the vehicle can endanger passengers with pacemakers or even damage magnetic data storage devices such as hard drives and credit cards; 
- The vehicle needs auxiliary wheels when driving at low speeds;

- Cost per km system still considered very high;

- There is a considerable cost and inconvenience of having to keep Celsius.

refrigerated superconducting magnets at 5 Kelvin at -268.15 degrees

\section{The advantages of the Inductrack System are:}

- Uses permanent magnets and does not use superconducting magnets;

- No need for sensors to control levitation;

- It does not give a strong magnetic field to passengers.

\section{The disadvantages of the EDS System are:}

- It is a recent system, there is no real-scale experimental line, so it can not be ascertained whether it will be effective compared to the other two magnetic levitation systems.
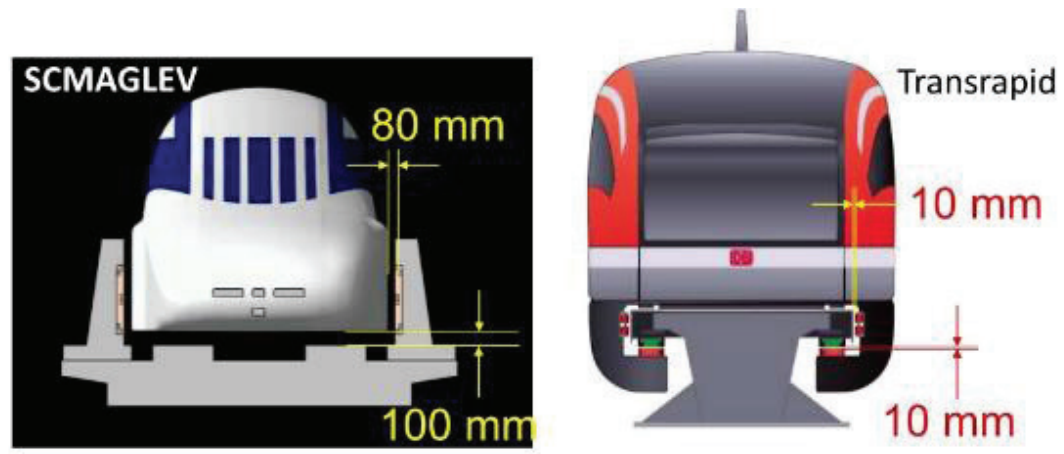

Fig. 21. Comparative distances between SCMAGLEV and Transrapid

(Source: Central Japan Railway Company, Yoshiyuki Kasai, 2012)

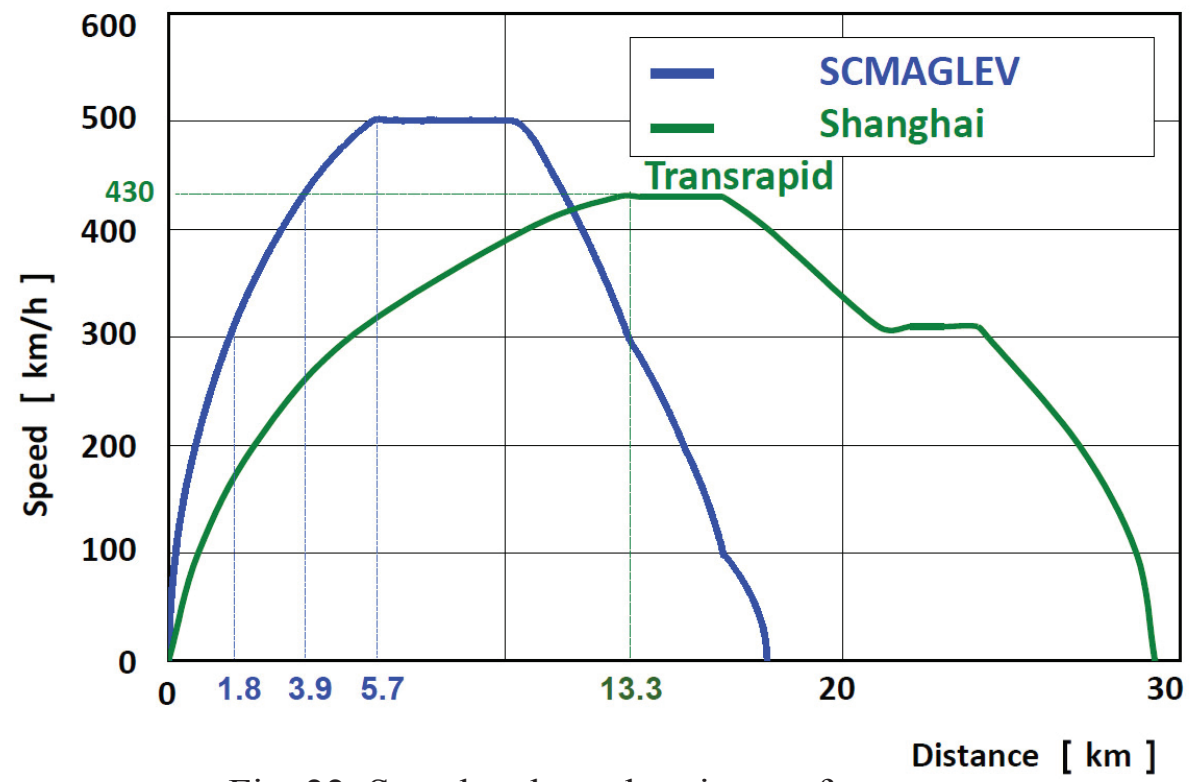

Fig. 22. Speed and acceleration performance

(Source: Central Japan Railway Company, Yoshiyuki Kasai, 2012) 
The following image illustrates the differences between the Transrapid and SCMAGLEV relative to the distances of the vehicle to the rail.

The following image shows the differences that exist in the maximum speed between the Transrapid system and SCMAGLEV, as well as the distance that each system takes to reach the maximum speed and the distance that it takes to stop.

\section{NEW PROPOSED SYSTEM (PRODYNAMIC)}

The objective in this article is to present a possible evolution to the EMS system. As already mentioned, both the conventional railway system and the highspeed railway network present physical limitations that are intrinsic to the wheel-rail contact system, among others, the lack of friction and the costs of maintenance.

The proposed system is very similar to the existing magnetic levitation systems, the existing Transrapid system and the Japanese SCMaglev system.

This presented system was conceived by an ISEP team (teachers and students) and could be registered as a new/different approach to existing magnetic levitation systems.

The following proposal is an improvement which consists of a theoretical possibility that the rail can achieve through magnetism, apply a rotation to the required vehicle in a curve and adjust that rotation to the speed at which it travels. This system represents a new concept, an evolution and putting into practice has several advantages. The main advantage is that the construction of the rail is uniform and does not require static superelevation or cant, making the manufacture and installation of the rail much more economical.

In order for this system to work, it is suggested that a further magnetic field be introduced on the track, in addition to the propellers and guidance, which is to rotate the vehicle in a curve and to replace the superelevation of the conventional rail. This rotation in addition to the existing advantages in the construction process, also has the advantage of the vehicle adjusting the rotation according to the speed at which it travels, ensuring the best comfort for the passengers. The proposed system has, as with other magnetic levitation systems, lateral guiding forces, lift forces, propulsion forces, but also rotational forces that are only required in a curve. These magnetic forces are arranged on the rail which interact with the magnets in the vehicle as can be seen in the following figure.

The following image shows a longitudinal diagram of the rail and how it can induce rotation through a magnetic field. The rail is segmented to induce a different rotation in each section according to the speed that the vehicle is traveling in a curve.

Therefore, the construction of the rail becomes much simpler in its development in curve and achieves a reduction in the costs of execution and 


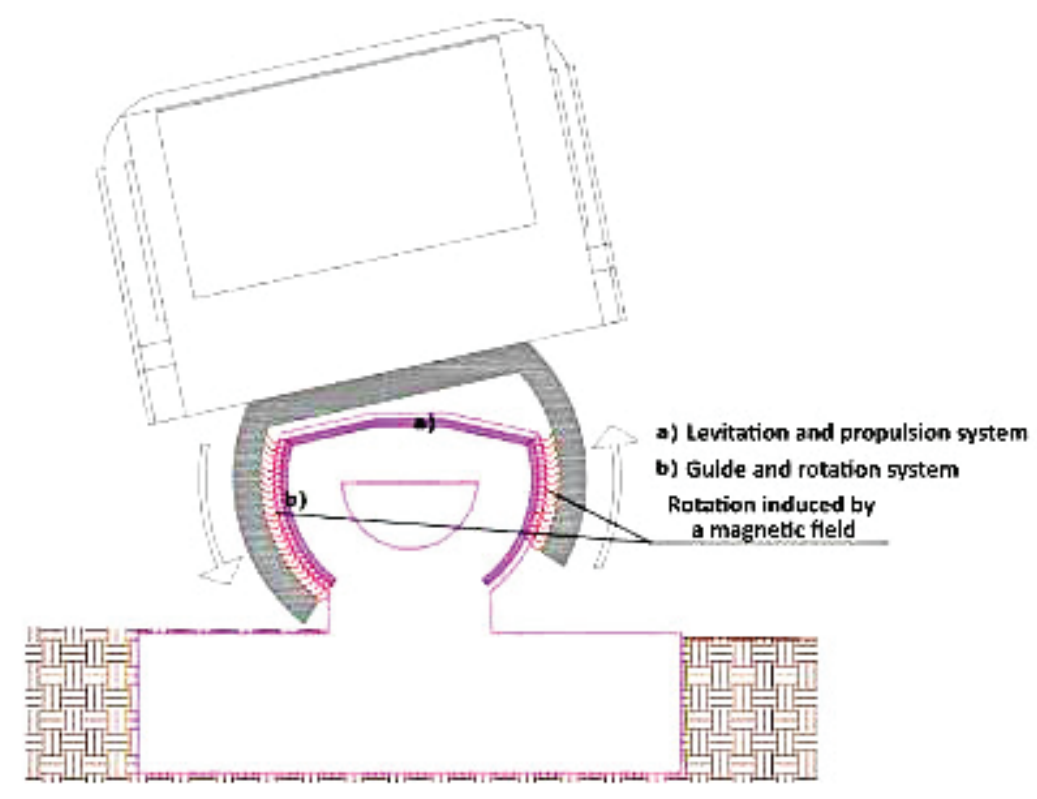

Fig. 23. Rotation scheme of the vehicle induced by a magnetic field

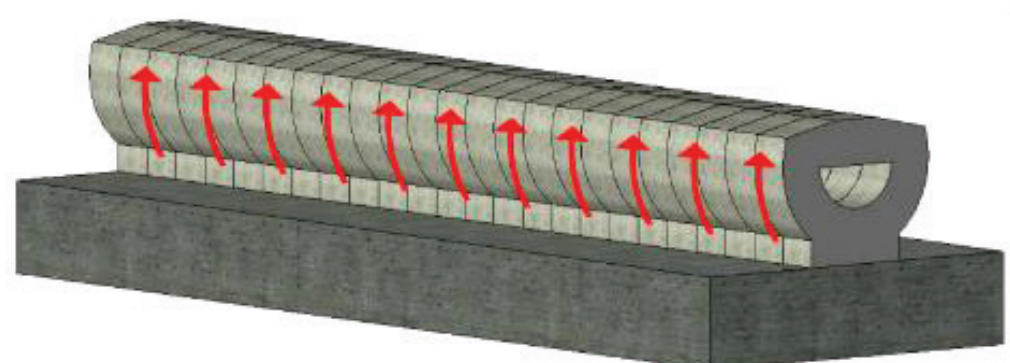

Fig. 24. Longitudinal scheme of the rail and the induced magnetic field, to generate the rotation

design, thus making more feasible the implementation of the magnetic system. For this system two hypothetical types of magnetic levitation are presented which theoretically are possible to conceive and represent an evolution to the systems currently in use and in tests. The following image shows one of the possible systems to be proposed, now called System 1.

The following image shows the other system to be proposed as system 2 . The systems proposed here are distinct and are an evolutionary variant of current magnetic levitation systems.

Systems 1 and 2 are distinct in the form of the rail and in the shape of the base of the vehicle. The system 1 has a «claw» that surrounds the rail, while in system 2 the rail engages the vehicle as can be seen in the image. However, both systems use an identical levitation system. The technology proposed for the vehicle to turn in a curve is identical for both systems, so there is no need to superelevate in either of the two proposed systems. This is achieved in the construction of this 


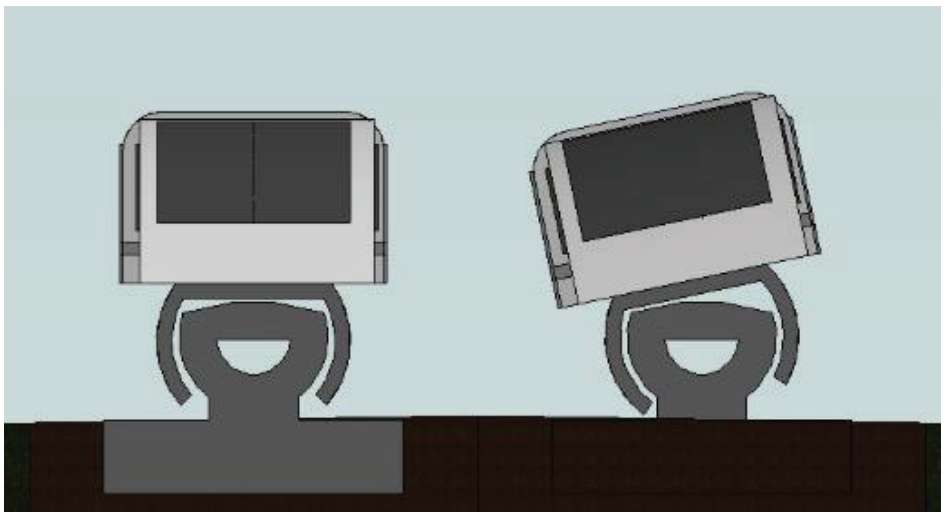

Fig. 25. Alternative magnetic levitation system 1 proposed

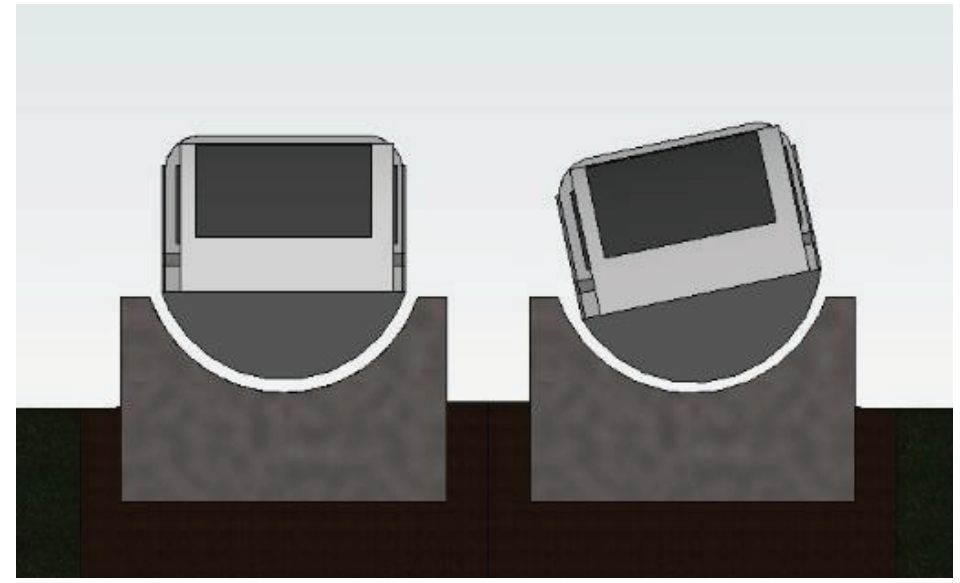

Fig. 26. Alternative magnetic levitation system 2 proposed

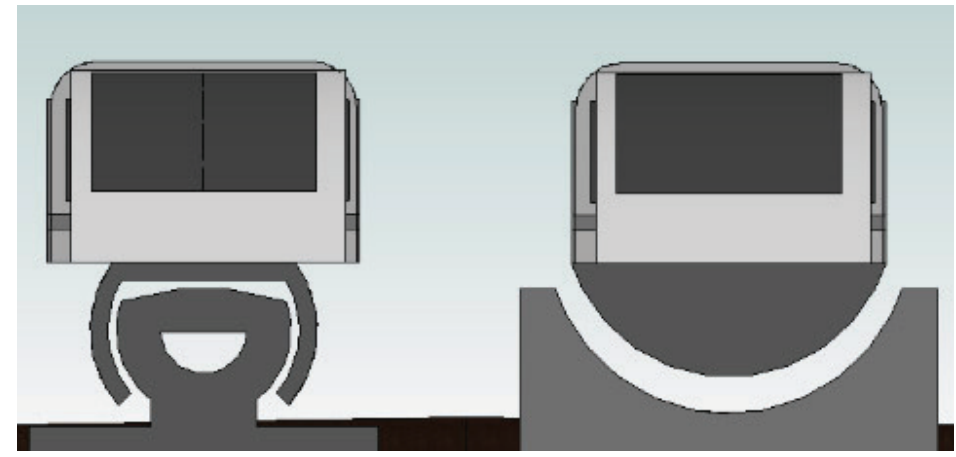

Fig. 27. ProDynamic system 1 and system 2

type of system, a productivity continues in both straight and curved, in addition the system guarantees in curve the best relation between the rotation and the speed to which it moves, guaranteeing the best possible adaptive comfort for passengers.

In the following image also it is verified that the upper part of the vehicle is equal in both systems, changing only the lower part, being able to have interoperability of infrastructure. 


\section{SYSTEM 1 - PRODYNAMIC}

System 1 results in part from a variation of the existing system in the German Transrapid. This system also has a form of grip that fits on the rail, such as Transrapid.

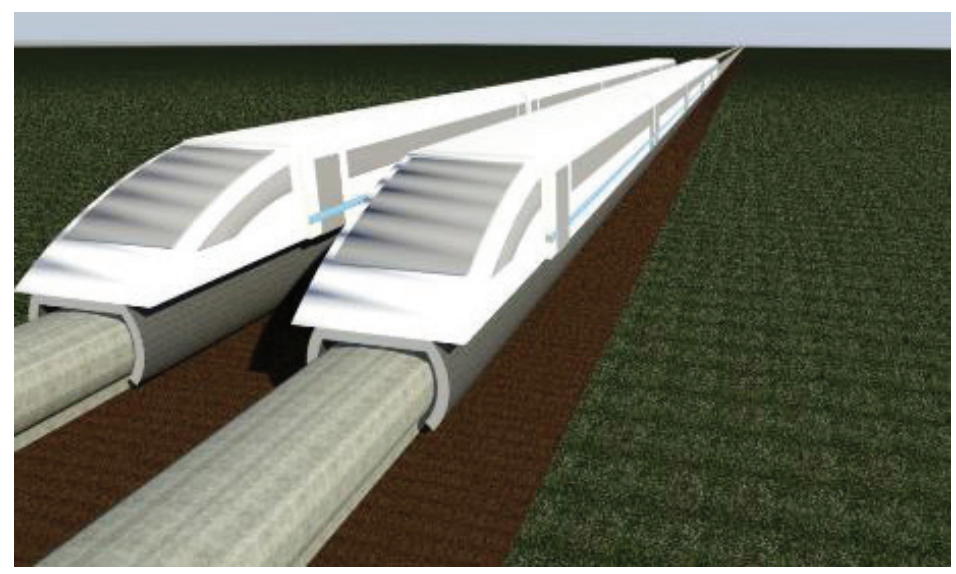

Fig. 28. Virtual image ProDynamic system 1 proposed

In the following image we can verify the similarities between the two systems, but the great difference between them consists in its curve layout, in which the proposed system does not need static superelevation. This system can rotate on the rail and apply the angle that it needs according to the speed while travelling, ensuring a greater comfort to the passengers and facilitating the constructive process with respect to the construction of the curved rail. The proposed system adapts to the speed, looking for the ideal rotation position.

The differences are essentially related to the rail and to the way the vehicle moves on the track. In the following image we can verify the constructive characteristics of the rail of this system in its longitudinal development.

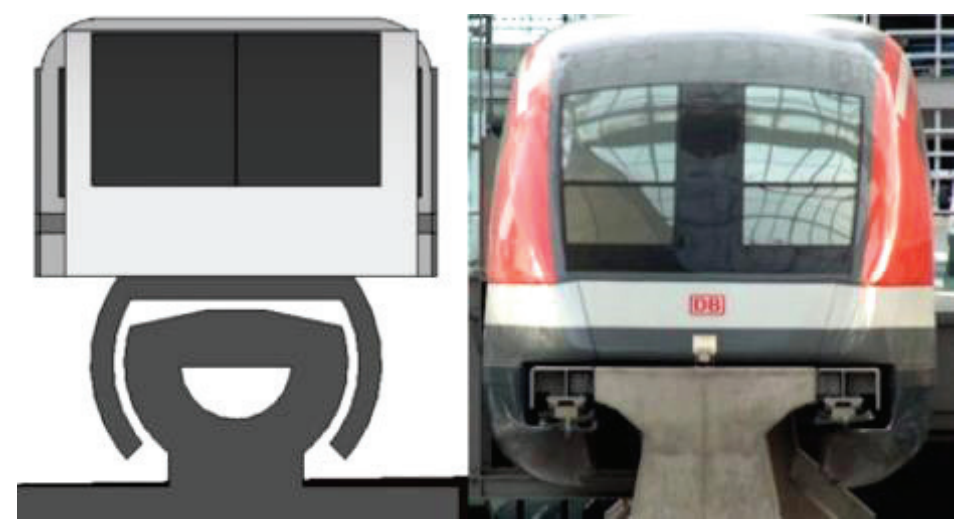

Fig. 29. Comparison between the proposed system 1 (ProDynamic) and the Transrapid system 


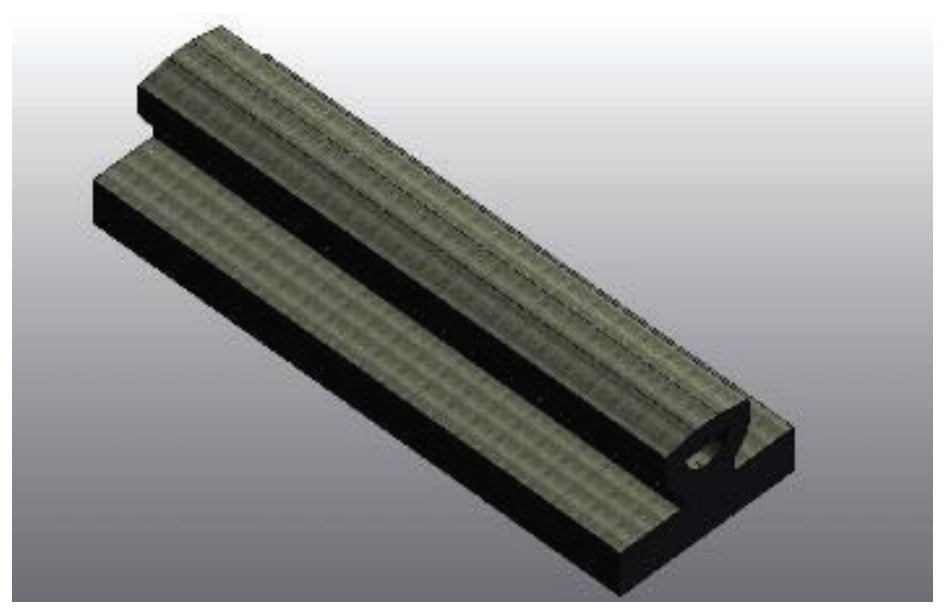

Fig. 30. Path to be used in system 1 proposed

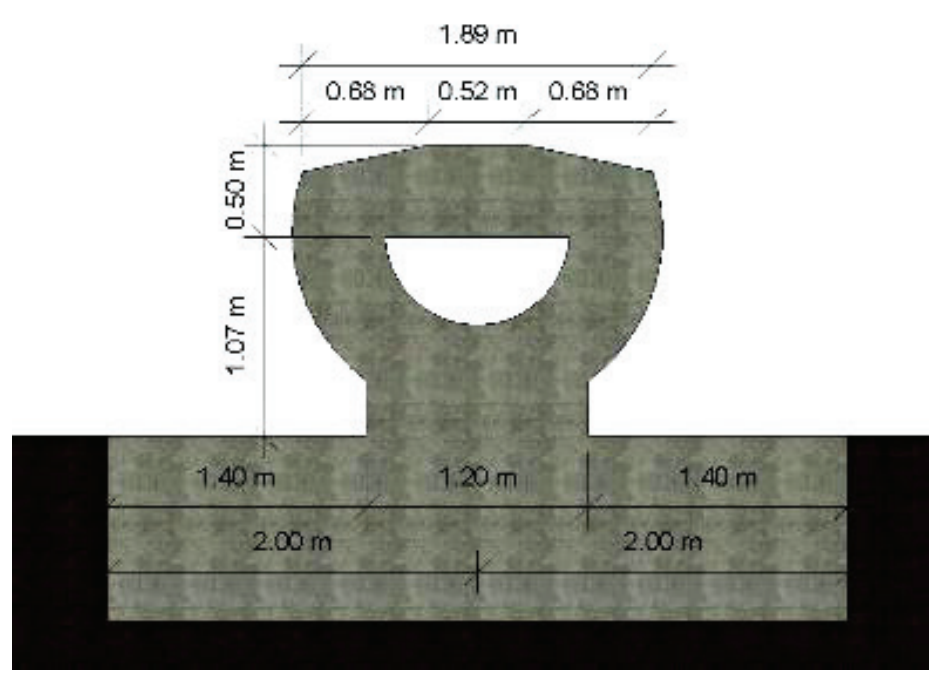

Fig. 31. Cross section of the proposed system 1 rail

The rail configuration has a cylindrical base that allows the vehicle to roll over the rail. This configuration assumes that it will be possible, through the induction of another magnetic field, to cause the vehicle to rotate on the track. With this suggestion there is a need for a third magnetic force that applies a necessary force to the vehicle so that it rotates the necessary one according to the speed in which it moves in curve, diminishing the effect of the discomfort due to the centrifugal acceleration. In addition, this magnetic field also requires constant computer control so that this rotation remains within the safety limits. The following figure shows a cross section of the rail of this system and its possible dimensions.

The vehicle has dimensions like those of the Transrapid, where they only differ in the lower area of the vehicle where it interacts with the rail. 


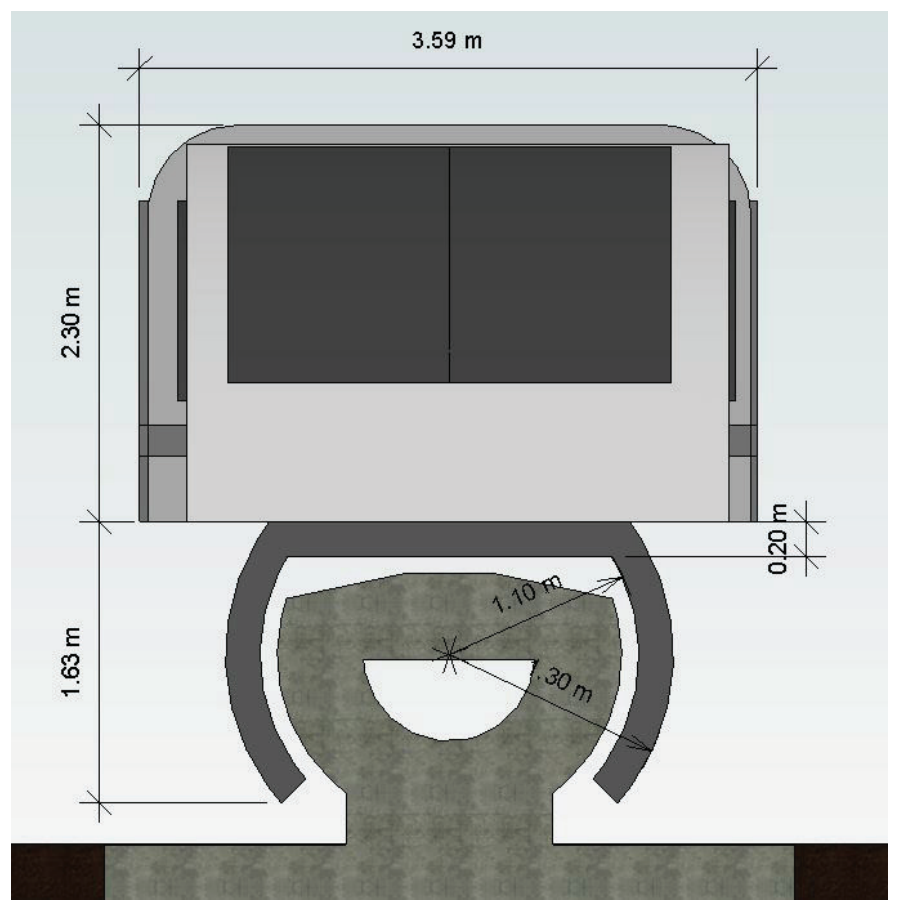

Fig. 32. Transversal perspective of the system 1 vehicle

Shown in next figure is a longitudinal perspective view of the vehicle of system 1 and its dimensions. The vehicle is composed of carriages of $15 \mathrm{~m}$ length, or in the case of the compositions of the extremities these are about $20 \mathrm{~m}$ in length.

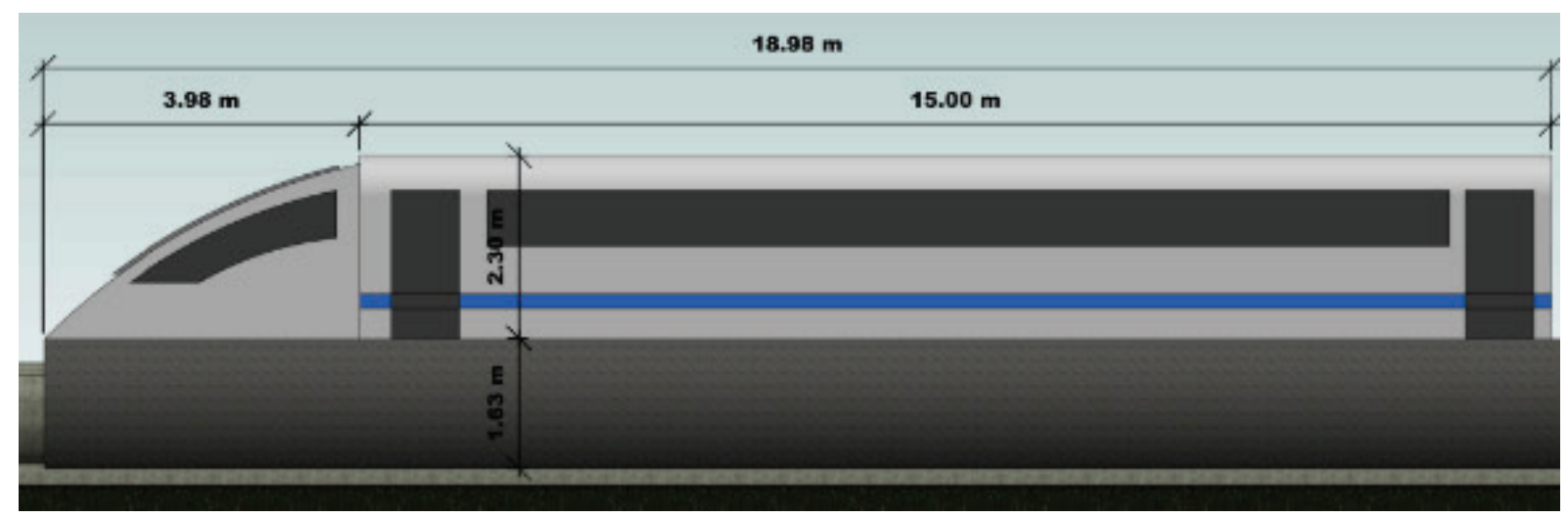

Fig. 33. Longitudinal perspective of the vehicle of the system 1

The configuration of the rail has characteristics that allow the vehicle to turn on the curved rail, with no static cant. In the following image it can see how the vehicle rolls on the rail at a maximum angle of $12^{\circ}$, but may be higher depending on the geometry defined for the claws and base support. 


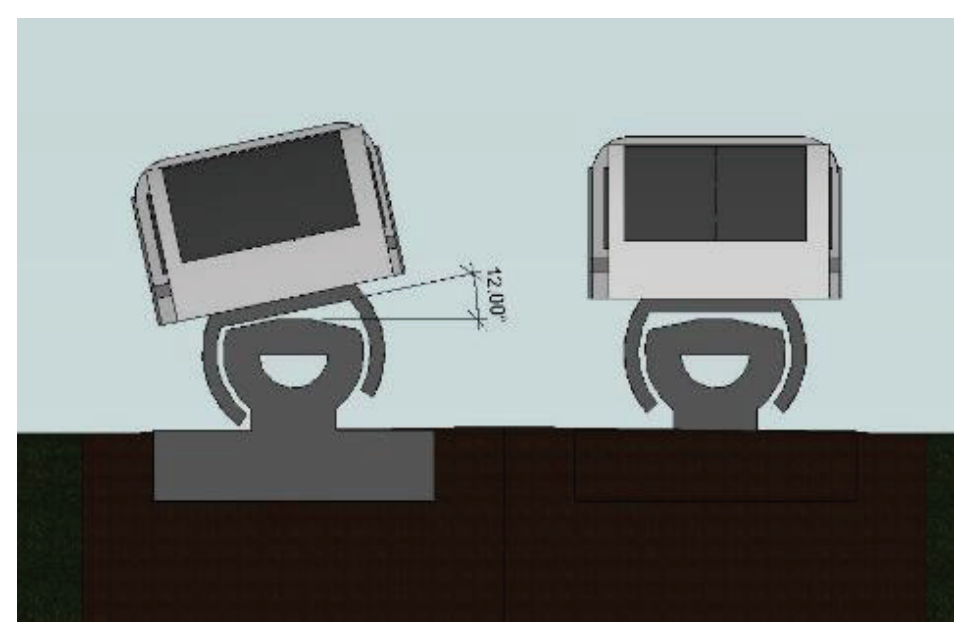

Fig. 34. Rotation of the vehicle in system 1 proposed

For the moment, this system has a maximum configuration of $12^{\circ}$ and manages to guarantee an optimum relation between the speed at which the vehicle moves and the necessary rotation for that speed, always maintaining the ideal comfort for the passengers. The comfort of the passengers depends on the speed at which the vehicle is traveling, the radius of the curve and the inclination of the vehicle. To minimize the effect of centrifugal acceleration it is necessary that there is an ideal relationship between these three factors. The acceleration can be quantified by the simple following expression:

$$
a_{c}=\frac{v^{2}}{R},
$$

where:

$$
\begin{gathered}
a_{c}-\text { centrifugal aceleration }\left(\mathrm{m} / \mathrm{s}^{2}\right) \\
v-\operatorname{velocity}(\mathrm{m} / \mathrm{s}) \\
R-\text { radius of the curve }(\mathrm{m})
\end{gathered}
$$

The centrifugal acceleration for rail has register values for passenger comfort up to a maximum of $1.5 \mathrm{~m} / \mathrm{s}^{2}$. Above this value, passengers begin to feel some discomfort. For the calculation of the centrifugal acceleration it was considered the condition of comfort limit conditioning for the passengers, the high speed and in curve. This analysis consists of moving the vehicle to an operating speed of $450 \mathrm{~km} / \mathrm{h}$ with a minimum radius of curvature of $4400 \mathrm{~m}$. In the following expression the value of $\mathrm{a}_{\mathrm{c}}$ can be verified: 


$$
a_{c}=\frac{\left(\frac{(450 \times 1000)}{3600}\right)^{2}}{4400}=3,551 \mathrm{~m} / \mathrm{s}^{2}
$$

It should be noted, however, that to obtain the correct comfort value for passengers, the calculation of unbalanced acceleration is used, which consists of subtracting the component from the centrifugal acceleration with the acceleration component due to the weight of the vehicle. These acceleration components are schematically shown in the following figure.

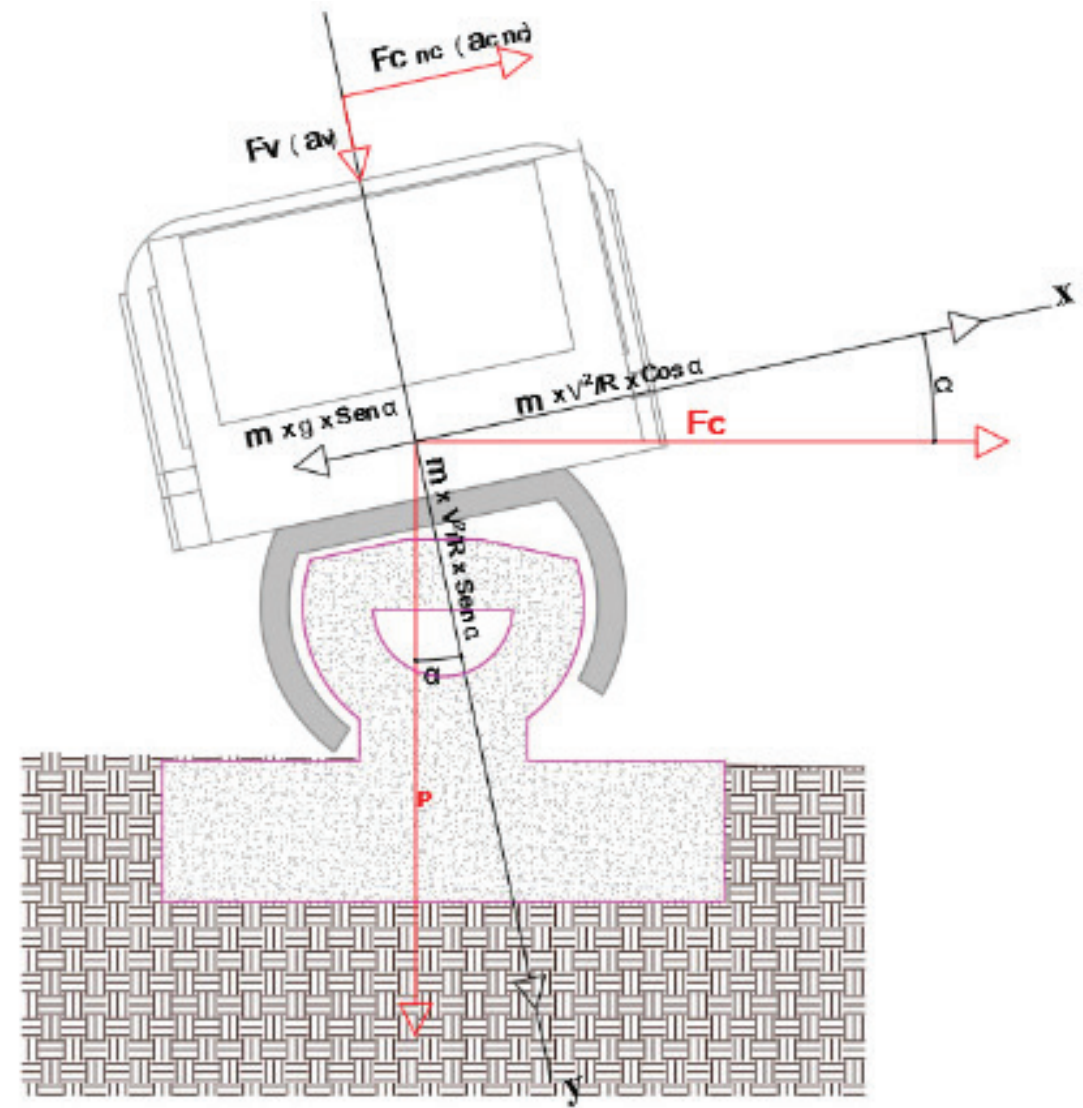

Fig. 35. Schematic of the forces acting (acceletarion directions) on the vehicle

The calculation of the acceleration component due to the weight of the vehicle for the situation depicted in the image above is obtained by expression (3).

$$
g \times \sin (\alpha)=9,8 \times \sin \left(12^{\circ}\right) \rightarrow 2,037 \mathrm{~m} / \mathrm{s}^{2}
$$

The calculation of the centrifugal acceleration component for the situation represented at an angle of $12^{\circ}$ is obtained by expression (4). 
According to the result obtained, it possible to calculate the component of the centrifugal acceleration for the situation in analysis as can be verified in expression (4).

$$
\frac{v^{2}}{R} \times \cos \alpha=3.551 \times \cos \left(12^{\circ}\right) \rightarrow 3.474 \mathrm{~m} / \mathrm{s}^{2}
$$

Using the values of expression (3) and expression (4) of the situation under analysis one obtains the unbalanced acceleration as can be seen in the next expression (5).

$$
a c n c=3.474-2.037 \rightarrow 1.437 \mathrm{~m} / \mathrm{s}^{2}
$$

Thus, a value of $1.437 \mathrm{~m} / \mathrm{s}^{2}$, which is within the comfort values for passengers, was obtained up to $1.5 \mathrm{~m} / \mathrm{s}^{2}$. In the following figure for an operating speed of $450 \mathrm{~km} / \mathrm{h}$, limiting the vehicle's rotation to $12^{\circ}$, only comfort values with radii above $4300 \mathrm{~m}$ are achieved for unbalanced lateral acceleration.

The following image shows the possible inclination (rotation) for the operating speeds and the unbalanced acceleration for each speed considering a radius of $4440 \mathrm{~m}$. It is verified that all the values of the lateral unbalanced acceleration are within the comfort parameters. The acceleration depends on the

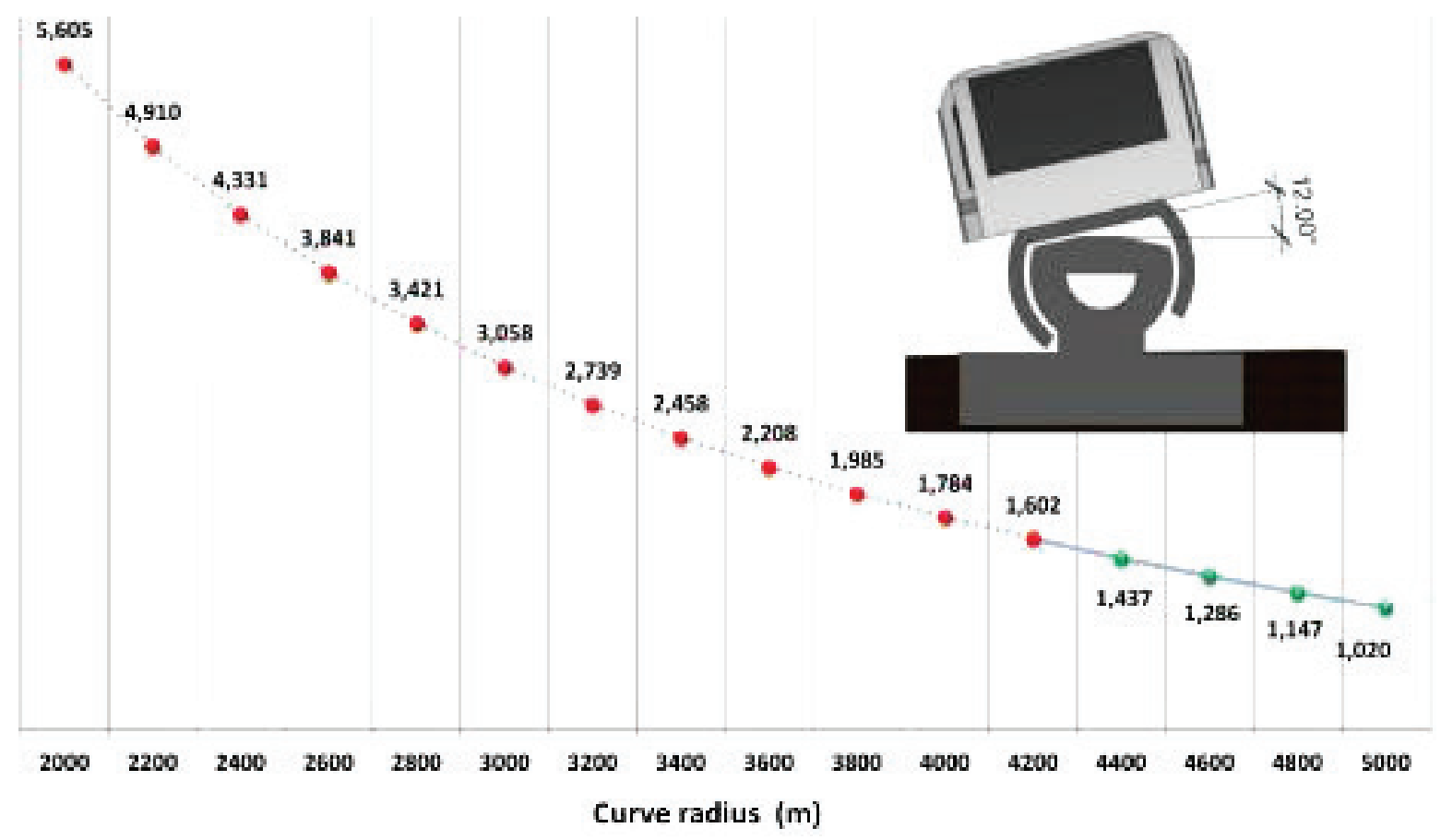

Fig. 36. Uncompensated acceleration for a speed of $450 \mathrm{~km} / \mathrm{h}$ with $12^{\circ}$ inclination versus several curve radius 


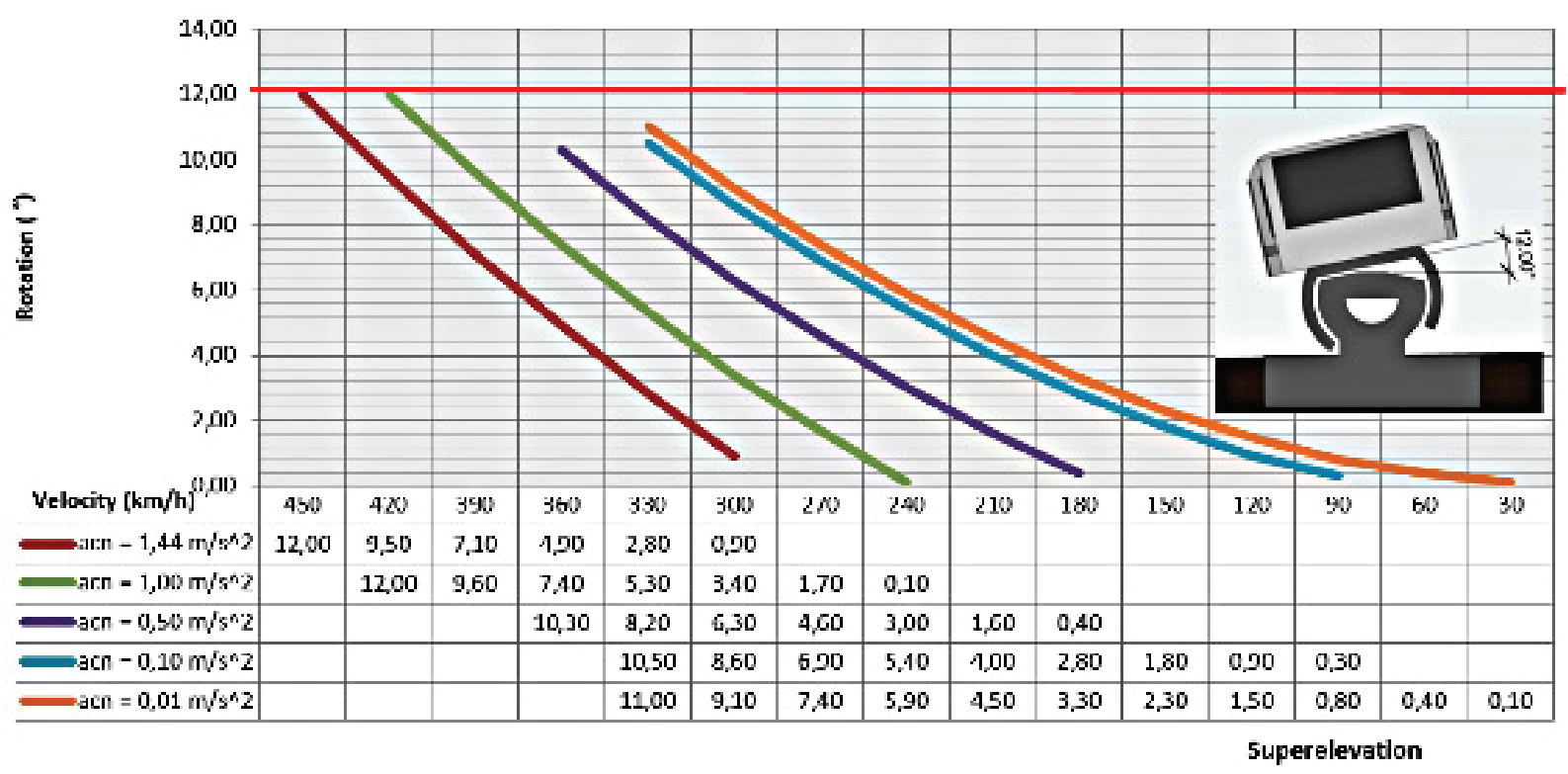

Fig. 37. Possible inclinaton (rotation) for operating speeds considering a constant radius of $4400 \mathrm{~m}$

speed and therefore these values can always be adjusted in a curve, according the inclination and the comfort values admissible for the acceleration.

In this way, it is understood that this system guarantees a good relation between the degree of inclination, operational speed and comfort of passengers, guaranteeing a simplicity in the construction especially in a curve where this system does not have any type of superelevation in curve. The great advantage is that the work of rail construction is constant, either in a curve or straight, reducing construction costs significantly.

\section{SYSTEM 2 - PRODYNAMIC}

System two results from a variation of the existing system in the Japanese MXL01.

This system has a circular shape identical to the "U" shape that fits over the rail, such as SCMaglev. In the following image the differences between the two systems can be identified.

As in system 1, system 2 differs from the SCMaglev system due to the rail and the way the vehicle is driven over the track. This system allows, such as the system 1 , that the vehicle turn on itself in a curve and does not require the existence of cant in the rail. This situation as in the system 1 involves the introduction of a magnetic force which rotates the vehicle in a curve according to the speed at which it travels. 


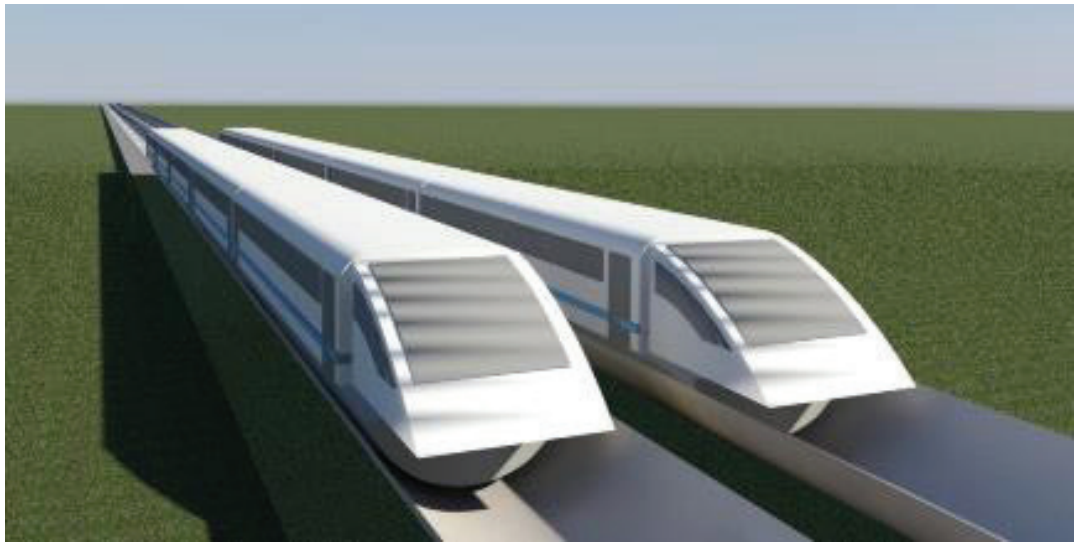

Fig. 38. System 2 - ProDynamic proposed

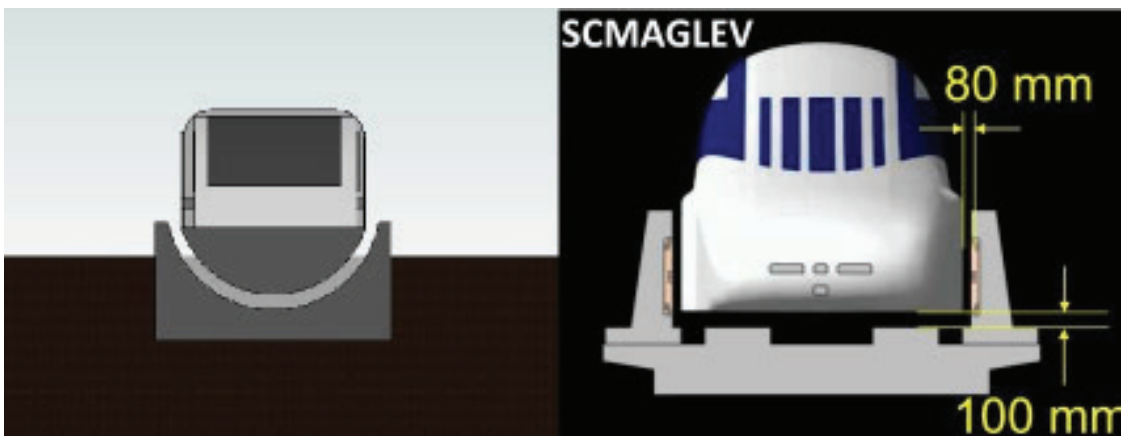

Fig. 39. System 2 - Comparison between the proposed system 2 and the SCMaglev system

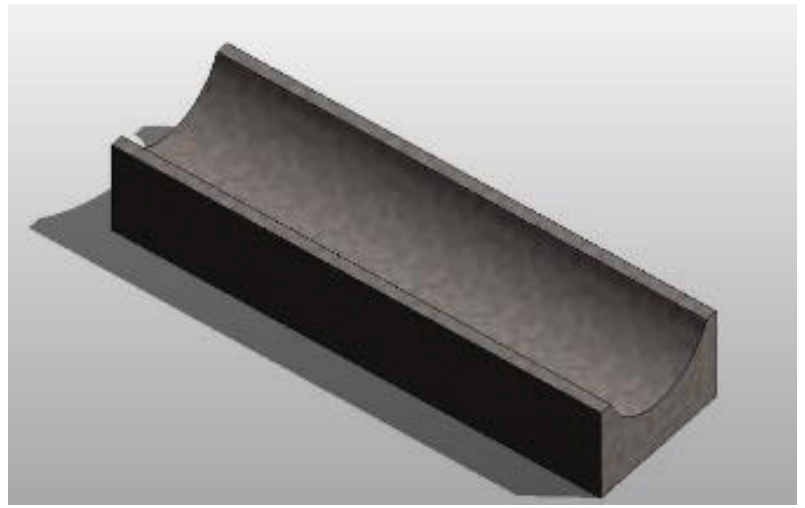

Fig. 40. Path to be used in system 2 proposed

The following image shows a cross section of the rail system proposed and its dimensions. As this track is continuous and always has the same geometric characteristics makes it very easy to build, and high-performance pre-fabrication can be used for a quick and economical solution. 


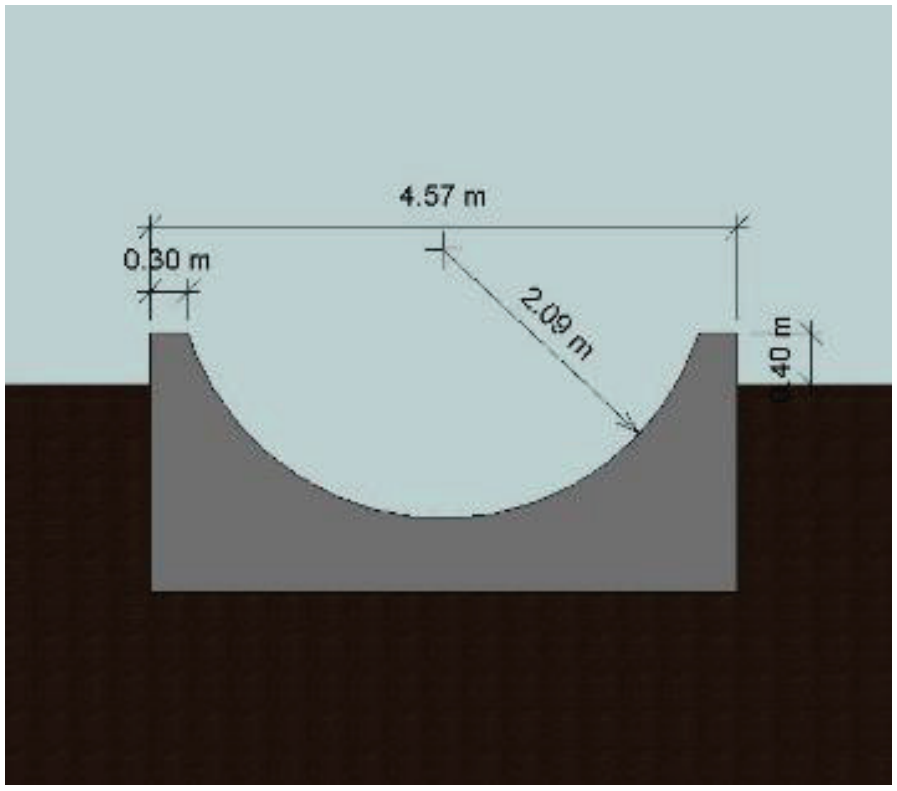

Fig. 41. Cross section of the proposed system 2 rail

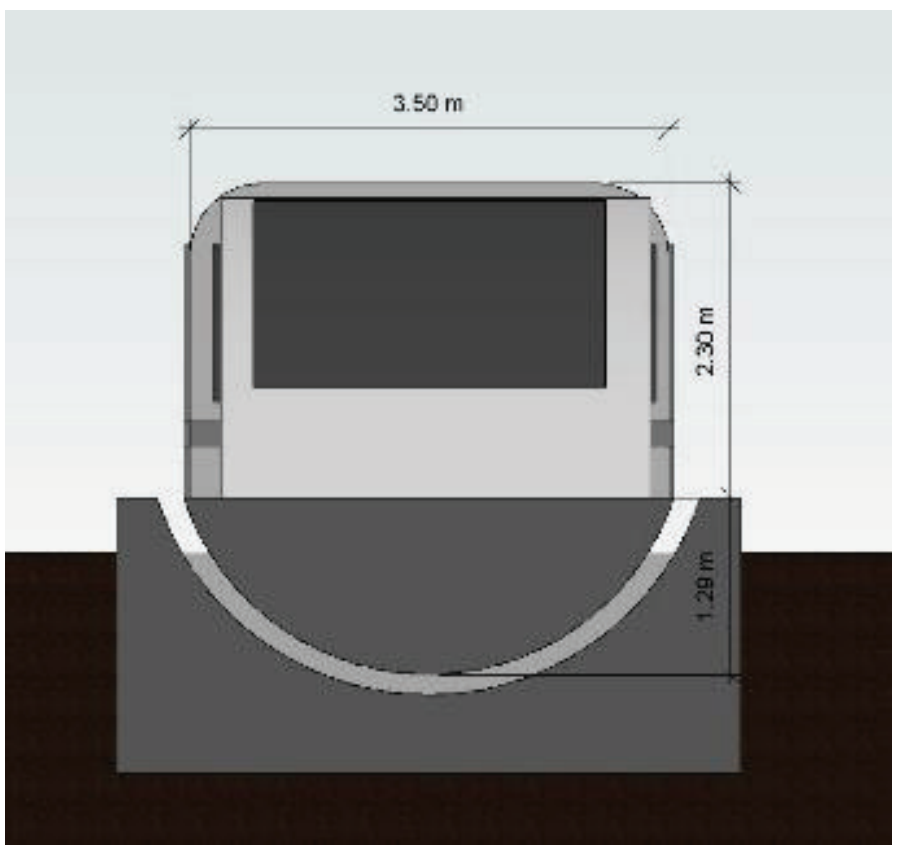

Fig. 42. Transverse perspective of system 2 vehicle

The following figure shows a cross-sectional view of the vehicle system 2 and its dimensions. As in system 1, system 2 is also like SCMaglev, differing only in the form of rail contact.

This system such as system 1 has a configuration that allows a maximum rotation of $12^{\circ}$. The system can guarantee an optimum relation between the speed at 


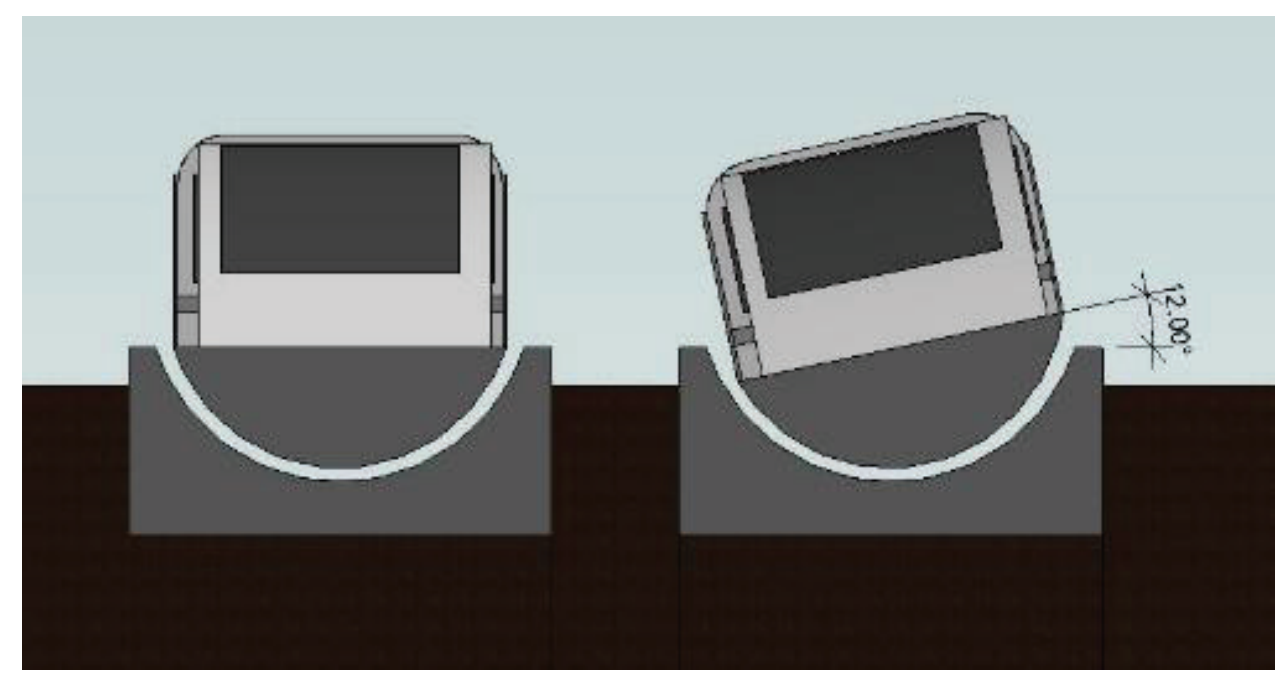

Fig. 43. Rotation of the vehicle in system 2 proposed

which the vehicle moves and the necessary speed for this speed, always maintaining the ideal comfort for the passengers.

The relationships between maximum operating speed, curved radius and slope of $12^{\circ}$ are identical to the expressions in system 1 . The minimum radius considered for this system was also $4400 \mathrm{~m}$, so the results are the same.

This system, such as system 1, guarantees a good relationship between the degree of inclination of the operating speed and the comfort of the passengers, due to its simplicity in the construction, especially in a curve where this system does not require any type of superelevation.

\section{CONCLUSION}

After analysis it can be stated that high-speed railway lines are expanding all over the world, with several countries showing an interest in implementing the system or renewing their railway lines. However, based on the research developed in this work, high-speed systems probably reached the apogee of their development, at least for commercial speeds exceeding $350 \mathrm{~km} / \mathrm{h}$. The rail wheel contact system has physical limitations and problems such as lack of friction, which can compromise the security of the system or prevent the correct operation of the system.

Magnetic levitation systems represent the ideal alternative or complement to the current high-speed lines of the railways, since they are a system where there is no physical contact with the rail, eliminating any type of friction, being a system faster, quieter, with lower maintenance costs and more efficient energy consumption. They exhibit greater acceleration and greater braking capacity, 
managing to overcome superior longitudinal slopes and do not have problems of insufficiency or of excessive cant, like in railways.

In addition to these advantages, the systems presented in this work have evolutionary proposals that allow to reduce costs, especially in the construction phase, considering that the system does not require static superelevation in a curve. The cant is associated with the vehicle and not with the track, allowing continuous construction and the possibility of using prefabricated elements. As the vehicle is turning in a curve due to the existence of a magnetic field, there is no need superelevation of the track, reducing construction times, such as tight topography controls and proper adjustments to ensure the necessary inclination. The rail induces to the vehicle magnetic fields that serves, transverse guiding, sustentation along the rail, propulsion and braking, and the rotation that substitutes the cant, also defined by superelevation. Rotation of the vehicle is only performed in a curve, and this rotation has the advantage of adjusting the speed of the vehicle, ensuring the best comfort for passengers. The rotation being adjustable in a curve is more advantageous than an existing fixed cant for the entire life of the infrastructure. The vehicle may, for various reasons, can need travel at a lower design speed and if this happens and if the speed is lower than the design speed, passengers may feel the opposite effect of centrifugal force and pass to feel the centripetal force. This phenomenon is more evident if the vehicle stops in a curve, generating a lot of discomfort in the passengers, in the case of the static cant. So, when the rotation is performed in real time, for a certain speed, this system guarantees a better degree of comfort and if by chance the vehicle stops in a curve this adjusts automatically, as if it were a straight line. This control of the rotation, guiding, sustentation, propulsion and braking is realized by electronic control that adjust in real time all the parameters, guaranteeing the safety and the comfort of the passengers.

During this work it was found that levitation systems still present a higher implementation cost than existing high-speed railroad systems. However, given the lower energy consumption and the low costs of maintaining magnetic levitation systems, it can be said that the investment made will rapidly have a return expected in comparison to the railway systems.

The systems referred to in this work present a series of challenges and, to physically check their operational possibility, it will be necessary to carry out a scale model of the vehicles and the rails, so that the rotation of the curved systems can be checked. Prior to the execution of this model, several experiments should be carried out to verify how their various magnetic fields will behave when the vehicle turns in a curve through the introduction of a new magnetic force and how can these be calibrated and balanced forces. If it is possible to verify the operation of the system, there will be a need to check and size the rails for the two systems 
proposed here. In addition to the sizing of rails, construction methods can also be developed for each type of system in order to guarantee the highest productivity and the lowest possible cost.

Rotation of the vehicle and locomotion through magnetism may have applicability in other sectors of the industry, as has happened with the application of magnetic levitation in some elevators that are currently being developed.

Therefore, the systems presented here are an improvement to the proposed systems, however, it will be necessary to form a multidisciplinary research team in its various areas of science, to plan, experiment, adjust the systems from the project to its applicability and operation, thus guaranteed a significant improvement to a system that is already innovative.

The ProDynamic system is in this sense to give a new technological boost when the vehicle describes curves, thus avoiding any system of static infrastructure superelevation in the rail, or even complex mechanical hydraulic systems in vehicles that creates inclination of the vehicle. It is a theoretical system that lacks technical viability analysis in terms of electromechanical.

In conclusive terms, it can be said that if this system is technically feasible, the civil construction of the rail will be much more economical when compared to the current systems and in terms of comfort in circulation, the phenomena of insufficiency or excess of cant cease to exist.

\section{References}

1. Henry B, Comstock. A Concise Illustrated History of the Development of Steam Traction. Greenberg Pub; 1993. ISBN-10: 0897783573

2. Clifford F, Bonnett J. Practical Railway Engineering. 2nd ed. Imperial College Press; 2005. ISBN 10: 1860945155

3. Čermák JR, Bartoň L, Spal P, et al. Overview of Magnetic Levitation Principles and their Application in Maglev Trains. Advanced Engineering. 2008;2(1):19-28. ISSN 1846-5900. [cited 2018 June 9]. Available at: https://ru.scribd.com/document/55198430/magnetictrolley-for-industrial-use

4. Zhou L, Shen Z. Progress in high-speed train technology around the world. Journal of Modern Transportation. 2011;19(1):1-6. doi: 10.1007/BF03325733

5. Sands BD. The Transrapid Magnetic Levitation System: A Technical and Commercial Assessment. The University of California Transportation Center CA 94720; no.183. [cited 2018 June 9]. Available at: http://www.maglev.ir/eng/documents/reports/IMT_R_16. pdf

6. Coelho A, Santos J, Soares A. A Via Ferroviária de Alta Versus Via de Levitação Magnética. Final dissertation, Project, ISEP, Porto, 2010

7. Siemens AG, Reserved AR. Shanghai Maglev Transrapid Technology. Propulsion Traveling Field, Trafikudvalget 2006. 
8. Thome R, Radovinsky A, Montgomery B. EDS Levitation and Guidance Using Sheet Guideways 2 Magplane System Description 1 Introduction Design Issues for Sheet Guideways. 1992, no. 617. [cited 2018 June 9]. Available at http://citeseerx.ist.psu.edu/ viewdoc/download?doi=10.1.1.487.561\&rep=rep1\&type=pdf

9. Hoburg JF, Post RF. A Laminated Track for the Inductrack System: Theory and Experiment [cited 2018 June 9] Available at: http://www.askmar.com/Inductrack/2004-10\%20 Inductrack\%20Laminated\%20Track.pdf

10. The ROMAG Maglev GRT Technology. [Internet]. Washington University; c2015-03. Available at: http://faculty.washington.edu/jbs/itrans/romag.htm

11. Bachelet E. [Internet]. The International Maglev Board; c2015-03. Available at: https:// www.maglevboard.net/en/facts/inventors/119-emile-bachelet

12. Transrapid Maglev in PR of China. [Internet]. The International Maglev Board; c201503. [cited 2018 June 9] Available at: https://www.maglevboard.net/en/pics/transrapidmaglev/35-maglev-board/pics/photos-images/116-transrapid-maglev-in-pr-of-china

13. Urban Magnetic Levitation (MAGLEV) Transit - Japanese HSST System. [Internet]. Washington University; c2015-03. [cited 2018 June 9]. Available at: https://faculty. washington.edu/jbs/itrans/hsstpage.htm

14. High Tech for "Flying on the Ground [Internet]. Transrapid International; c2015-06. [cited 2018 June 9]. Available at: https://www.ncl.ac.uk/media/wwwnclacuk/pressoffice/files/ pressreleaseslegacy/TRI_Flug_Hoehe_e_5_021.pdf

15. Carvalho FO. Elaboração de um Trilho MagLev Para Experimentos Didáticos. [Internet]. Universidade Estadual de Campinas.; c2015-07. Available at: https://www.ifi.unicamp. br/ lunazzi/F530_F590_F690_F809_F895/F530_F590_F690_F895/F530_F590_F690_ F895_sem2_2005/Fabricio_Dirceu_F895_RF2.pdf(In Portuguese).

16. Maglev: A New Approach [Internet]. Askmar; c2015-08. Available at: http:/www.askmar. com/Inductrack/2000-1\%20Inductrack\%20Scientific\%20American.pdf

\section{Information about the authors:}

Jacob Ângelo, Master degree Specialist, Adjunct Professor;

Dr. António Bernardino de Almeida str., 431, 4249-015 Porto, Portugal;

ORCID: 0000-0002-0998-4101;

E-mail: amj@isep.ipp.pt, angelo.jacob@civitrace.com

Monteiro Nuno, Master degree Civil Engineering, Infrastructures;

ORCID: 0000-0003-2150-1528;

E-mail: nunomanuelmonteiro@gmail.com

\section{To cite this article:}

Jacob AMG, Monteiro NMV. A New Concept of Superelevation in Magnetic Levitation Prodynamic. Transportation Systems and Technology. 2018;4(4):77-111. doi: 10.17816/ transsyst20184477-111 